\title{
Novel Subcarrier-Allocation Schemes for Downlink MC DS-CDMA Systems
}

\begin{abstract}
4 Abstract-This paper addresses the subcarrier allocation in 5 downlink multicarrier direct-sequence code-division multiple ac6 cess (MC DS-CDMA) systems, where one subcarrier may be as7 signed to several users who are then distinguished from each other 8 by their unique direct-sequence spreading codes. We first analyze 9 the advantages and shortcomings of some existing subcarrier10 allocation algorithms in the context of the MC DS-CDMA. Then, 11 we generalize the worst subcarrier avoiding (WSA) algorithm to 12 a so-called worst case avoiding (WCA) algorithm, which achieves 13 better performance than the WSA algorithm. Then, the WCA al14 gorithm is further improved to a proposed worst case first (WCF) 15 algorithm. Furthermore, we propose an iterative worst excluding 16 (IWE) algorithm, which can be employed in conjunction with the 17 WSA, WCA, and the WCF algorithms, forming the IWE-WSA, 18 IWE-WCA, and the IWE-WCF subcarrier-allocation algorithms. 19 The complexities of these algorithms are analyzed, showing that 20 they are all low-complexity subcarrier-allocation algorithms. The 21 error performance is investigated and compared, demonstrating 22 that we can now be very close to the optimum performance 23 attained by the high-complexity Hungarian algorithm.
\end{abstract}

24 Index Terms-Multicarrier, DS-CDMA, MC DS-CDMA, 25 OFDMA, LTE/LTE-A, resource-allocation, subcarrier-allocation, 26 greedy, complexity.

\section{INTRODUCTION}

28 T $\mathrm{N}$ wireless communications, multicarrier signalings have 29 attracted wide attention as one of the promising candi30 dates for high speed broadband wireless communications. 31 In multicarrier systems, multicarrier modulation/demodulation 32 can be implemented with the aid of low-complexity fast 33 Fourier transform (FFT) techniques. When appropriately con34 figurated, some multicarrier schemes, such as orthogonal fre35 quency division multiple access (OFDMA) and orthogonal 36 multicarrier DS-CDMA, employ the capability to suppress 37 inter-symbol interference (ISI) [1], [2]. Furthermore, the mul38 ticarrier DS-CDMA (MC DS-CDMA), in which each sub39 carrier uses direct-sequence (DS) spreading, employs a high 40 number of degrees-of-freedom for high-flexibility design and 41 reconfiguration [2].

42 It is now well-known that exploiting the time-varying 43 characteristics of wireless channels is capable of signifi44 cantly enhancing the quality-of-service (QoS) of wireless com45 munication systems. Specifically, with the aid of dynamic 46 subcarrier-allocation to users, promising energy- and spectral-

Manuscript received October 10, 2013; revised March 28, 2014; accepted June 24, 2014. The associate editor coordinating the review of this paper and approving it for publication was M. C. Gursoy.

The authors are with the School of Electronics and Computer Science, University of Southampton, Southampton SO17 1BJ, U.K. (e-mail: js22g09@ ecs.soton.ac.uk; 1ly@ecs.soton.ac.uk).

Digital Object Identifier 10.1109/TWC.2014.2338853 efficiency can be attained by making use of the embedded 47 multiuser diversity [3]. Owing to its above-mentioned metrics, 48 subcarrier-allocation in broadband multicarrier systems, such 49 as in LTE/LTE-A OFDMA, now becomes highly important. 50 In literature, such as in [3]-[10], various subcarrier-allocation 51 algorithms have been proposed and studied for downlink 52 OFDMA systems and other multicarrier systems. Specifically, 53 the (unfair) greedy algorithm has been investigated in [4] 54 without considering the fairness, which aims at maximizing the 55 total sum rate of downlinks. By contrast, in [5], [6], the (fair) 56 greedy algorithm has been studied, when fairness is taken into 57 account, making each user select the best subcarrier(s) from the 58 available subcarriers. However, in terms of reliability, the users 59 allocated the subcarriers at the late stages of the fair greedy 60 algorithm often have poor performance. In order to circumvent 61 the shortcomings of the fair greedy algorithm, in [7], a worst 62 subcarrier avoiding (WSA) algorithm has been proposed for 63 subcarrier-allocation in the downlink OFDMA and frequency 64 division multiple access (FDMA) systems. The studies in [7] 65 demonstrate that the WSA algorithm can effectively avoid 66 assigning users the subcarriers of the poorest channel qualities, 67 and can hence attain higher reliability than the fair greedy algo- 68 rithm. In subcarrier-allocation, the Hungarian algorithm [11] is 69 recognized the optimum algorithm in the sense of maximum 70 reliability, which has been investigated, for example, in [7], 71 [10]. However, the Hungarian algorithm is of high complexity 72 for implementation in the OFDMA systems with a high number 73 of subcarriers supporting a high number of users.

In LTE/LTE-A downlink OFDMA systems, the number of 75 subcarriers is usually very high, which is up to 2048, and 76 the number of users supported may also be very high. These 77 characteristics generate some problems, such as, the PAPR 78 problem, and may prevent schedulers from employing the 79 optimum or even some promising sub-optimum subcarrier- 80 allocation schemes, due to their complexity constraint. As 81 the complexity of the optimum or sub-optimum subcarrier- 82 allocation algorithms is mainly dependent on the number of 83 subcarriers, reducing the number of subcarriers may effectively 84 decrease the operation complexity of these algorithms. It is 85 well-known that, owing to the employment of DS spreading, the 86 MC DS-CDMA can use a significantly lower number of subcar- 87 riers than the multicarrier schemes, such as the OFDMA, which 88 do not employ DS spreading. Furthermore, MC DS-CDMA 89 employs the flexibility to configure its number of subcarriers 90 according to the frequency-selectivity of wireless channels, so 91 that each subcarrier experiences independent fading. In this 92 case, the number of subcarriers of MC DS-CDMA will be at the 93 order of the number of time domain resolvable paths of wireless 94 
95 channels and, hence, will usually be low [1]. Therefore, in MC 96 DS-CDMA, the relatively high-complexity optimum or near97 optimum subcarrier-allocation algorithms may be employed in 98 order to achieve the best possible performance.

99 A range of researches [12]-[18] have been dedicated to the 100 field of resource allocation in the MC CDMA and MC DS101 CDMA systems. The allocations of transmission rate, subcar102 rier and power have been considered in MC-CDMA system 103 in [14] for minimizing the total transmission power when 104 given certain bit error rate (BER) requirements. The authors 105 of [16], [17] have compared the capacity performance of the 106 MIMO-OFDMA and MIMO-MC-CDMA systems, when adap107 tive power allocation is employed. In [13], adaptive allocations 108 of subchannel, power and alphabet size have been addressed in 109 a distributed MC DS-CDMA system, in order to minimize the 110 transmit power under the constraint of packet rate.

111 Against the background, in this contribution, we study the 112 subcarrier-allocation in MC DS-CDMA systems. First, some 113 representative algorithms, including the greedy-family algo114 rithms, WSA algorithm, etc., are introduced to and studied in 115 association with the MC DS-CDMA systems. Then, a range 116 of subcarrier-allocation algorithms aiming at maximizing the 117 reliability of downlink MC DS-CDMA systems are proposed. 118 Furthermore, we propose a scheme, namely iterative worst ex119 cluding (IWE) scheme, which allows the proposed subcarrier120 allocation algorithms to achieve even better performance. In 121 this paper, the BER performance of the MC DS-CDMA systems 122 employing various subcarrier-allocation algorithms is investi123 gated, when assuming that subcarrier channels experience inde124 pendent fading. Our simulation results reveal that the proposed 125 algorithms may significantly outperform the existing subopti$126 \mathrm{mal}$ algorithms. Furthermore, the IWE scheme is effective for 127 further improving the BER performance of some subcarrier128 allocation algorithms.

129 The rest of the paper is organized as follows. Section II 130 introduces the system model and gives the main assumptions. 131 Section III states the principles of the proposed subcarrier132 allocation algorithms. Section IV discusses some existing 133 subcarrier-allocation algorithms and details the proposed al134 gorithms. Section V introduces the IWE scheme. Section VI 135 analyzes and compares the complexity of the considered 136 subcarrier-allocation algorithms. Section VII provides the BER 137 results and, at last, conclusions are summarized in Section VIII.

\section{System Models}

139 We consider a downlink MC DS-CDMA system which con140 sists of one base station (BS) communicating with $K$ mobile 141 users. We assume that each of the communicating terminals, 142 including BS and $K$ mobile users, employs one antenna for 143 signal receiving and transmission. Signals transmitted from BS 144 to mobile users are MC DS-CDMA signals using time (T)145 domain DS spreading [1] and the spreading factor is expressed 146 as $N$. For clarity, the variables and notations used in this paper 147 are summarized as follows:

$148 K \quad$ Number of mobile users;

$149 \mathcal{K} \quad$ Set of user indexes, defined as $\mathcal{K}=\{0,1, \ldots, K-1\}$;

$150 N \quad$ Spreading factor of DS spreading;
$M \quad$ Number of subcarriers of MC DS-CDMA systems; 151 $\mathcal{M}$ Set of subcarrier indexes, defined as $\mathcal{M}=152$ $\{0,1, \ldots, M-1\}$;

$h_{k, j} \quad$ Channel gain of subcarrier $j$ of user $k$; $\quad 154$

$C \quad(N \times K)$-dimensional spreading matrix with columns 155 consisting of the spreading sequences taken from a 156 $(N \times N)$ orthogonal matrix. Note that, some columns 157 of $\boldsymbol{C}$ may be the same in the case of $K>N$. In this 158 case, the corresponding users are operated on different 159 subcarriers;

$\mathcal{F}_{j} \quad$ Set of indexes for up to $N$ users assigned to subcarrier $j ; 161$

$|\mathcal{F}| \quad$ Cardinality of the set $\mathcal{F}$, representing the number of 162 elements in set $\mathcal{F}$;

$P_{k} \quad$ Transmission power for user $k$; 164

$P \quad$ Total transmission power of BS, $P=\sum_{k \in \mathcal{K}} P_{k} ; \quad 165$

$A_{k, j} \quad$ Channel quality of subcarrier $j$ of user $k, A_{k, j}=166$ $\left|h_{k, j}\right|^{2} /\left(2 \sigma^{2}\right)$, where $\sigma^{2}=1 /\left(2 \bar{\gamma}_{s}\right)$ denotes the single- 167 dimensional noise power at a mobile user and $\bar{\gamma}_{s}$ denotes 168 the average signal-to-noise ratio (SNR) per symbol.

169

In this paper, we assume that each user is allocated one 170 spreading code of one subcarrier. Consequently, we have 171 $\bigcup_{j \in \mathcal{M}} \mathcal{F}_{j}=\mathcal{K}, \mathcal{F}_{j} \cap \mathcal{F}_{i}=\emptyset$ for $i \neq j$, and there are possibly 172 $N$ users sharing one subcarrier. Let us assume that the data 173 symbols to be transmitted by the BS to the $K$ mobile users 174 are expressed as $\boldsymbol{x}=\left[x_{0}, x_{1}, \ldots, x_{K-1}\right]^{T}$, where $x_{k}$ is the 175 data symbol to user $k$, which is assumed to satisfy $E\left[x_{k}\right]=0176$ and $E\left[\left|x_{k}\right|^{2}\right]=1$. Furthermore, let us assume that the $j^{\prime}$ th 177 subcarrier is assigned to user $k$. Then, considering that the $M 178$ subcarriers are orthogonal, the signal received by user $k$ from 179 the $j^{\prime}$ th subcarrier can be written as

$$
\boldsymbol{y}_{k}=h_{k, j^{\prime}} \boldsymbol{C}_{k} \boldsymbol{P} \boldsymbol{W} \boldsymbol{x}+\boldsymbol{n}_{k}
$$

where, in addition to the notations mentioned previously, $\boldsymbol{y}_{k} 181$ is a length- $N$ observation vector, $\boldsymbol{n}_{k}=\left[n_{k, 0}, \ldots, n_{k, N-1}\right]^{T}$ is 182 a length- $N$ noise vector at user $k$, while $C_{k}$ is a $(N \times K) 183$ matrix formed from $C$ by setting those columns corresponding 184 to the subcarriers different from the $k$ th user's subcarrier to 185 zero vectors, as the result of using orthogonal subcarriers. In 186 this paper, we assume that uplinks and downlinks are operated 187 in the time-division duplex (TDD) mode. Hence, an uplink 188 channel and its corresponding downlink channel can be as- 189 sumed to be reciprocal. In this way, the BS is capable of 190 obtaining the knowledge of all the $K M$ downlink channels and, 191 hence, it can preprocess the signals to be transmitted by setting 192 $\boldsymbol{W}=\operatorname{diag}\left\{w_{0}, w_{1}, \ldots, w_{K-1}\right\}$, where $w_{k}=h_{k, j^{\prime}}^{*} / \sqrt{\left|h_{k, j^{\prime}}\right|^{2}} 193$ and $(\cdot)^{*}$ denotes the conjugate operation. We assume that the 194 channel-inverse power-allocation scheme is employed and, in 195 (1), the power assigned to each user can be expressed in 196 matrix form as $\boldsymbol{P}=\operatorname{diag}\left\{P_{0}, P_{1}, \ldots, P_{K-1}\right\}$. Consequently, 197 after the despreading for user $k$ using its spreading code $c_{k}, 198$ the $k$ th column of $C$, it can be shown that the decision variable 199 generated by user $k$ is

$$
z_{k}=P_{k} \sqrt{\left|h_{k, j^{\prime}}\right|^{2}} x_{k}+n_{k}
$$

which yields the SNR $\gamma_{k}=P_{k}\left|h_{k, j^{\prime}}\right|^{2} \bar{\gamma}_{s}=P_{k} A_{k, j^{\prime}}$. Explic- 201 itly, when allocating user $k$ a subcarrier with higher channel 202 
203 quality $A_{k, j^{\prime}}$, it attains a higher SNR and hence a lower 204 error rate.

205 Note that the above considered MC DS-CDMA scheme can 206 be straightforwardly extended to the scenarios where each of 207 the users demands multiple data streams depending on the data 208 rate required by the user. In this case, let $q_{k}$ represent the 209 number of data streams of user $k(k \in \mathcal{K})$. Then, we have 210 the constraint of $\sum_{k \in \mathcal{K}} q_{k} \leq M N$ on the resource allocation, 211 meaning that the total number of data streams does not exceed $212 M N$ in order to avoid interference. In this extended MC DS213 CDMA system, if $q_{k} \leq N$, user $k$ can be assigned one subcar214 rier and its $q_{k}$ data streams can be supported by assigning the 215 user $q_{k}$ different spreading codes. By contrast, if $q_{k}>N$, then, 216 user $k$ may be assigned multiple spreading codes and multiple 217 subcarriers, in order to support the $q_{k}$ data streams.

218 Note furthermore that our MC DS-CDMA scheme represents 219 a generalized multicarrier scheme for studying resource alloca220 tion. First, when $N=1$, i.e., when there is no DS spreading, 221 the MC DS-CDMA scheme is reduced to the conven222 tional OFDMA. Correspondingly, we only require subcarrier223 allocation, but no code-allocation. Second, when given the total 224 bandwidth of a MC DS-CDMA system, there exists a trade-off 225 between the number of subcarriers $M$ and the spreading factor $226 N$, which determines the bandwidth of subcarriers. Hence, in 227 a MC DS-CDMA system, the number of subcarriers can be 228 reconfigured according to the communication environments, 229 so that each of the subcarriers experiences flat fading, while 230 different subcarriers experience relatively independent fading. 231 Specifically, when operated in an environment where fading 232 is highly frequency-selective, the system may be configured 233 with a relatively high number of subcarriers but a relatively 234 low spreading factor, in order to guarantee that all subcarriers 235 experience flat fading. By contrast, when the communication 236 environment becomes less frequency-selective, the system may 237 be reconfigured to use a smaller number of subcarriers but 238 a bigger spreading factor. Owing to the reduced number of 239 subcarriers and the increased bandwidth per subcarrier channel, 240 different subcarriers will experience less correlated fading, 241 the complexity of subcarrier-allocation can be reduced and, 242 furthermore, the PAPR problem can be mitigated.

\section{General Theory of Resource Allocation}

244 In the MC DS-CDMA system, where $M$ subcarriers are 245 employed to support $K$ users, when the power- and subcarrier246 allocation are aimed to maximize the system reliability, the 247 optimization problem can be described as

$$
\begin{gathered}
\cup\left\{\mathcal{F}_{j}, P_{k}\right\}^{*}=\arg \min _{\cup\left\{\mathcal{F}_{j}, P_{k}\right\}}\left\{\bar{P}_{e}\right\} \\
=\arg \min _{\cup\left\{\mathcal{F}_{j}, P_{k}\right\}}\left\{\frac{1}{K} \sum_{k \in \mathcal{K}} \bar{P}_{e}^{(k)}\right\}, \\
\text { s.t. } \quad \cup_{j \in \mathcal{M}} \mathcal{F}_{j}=\mathcal{K}, \mathcal{F}_{j} \cap \mathcal{F}_{l}=\emptyset \text { for } j \neq l, \\
\sum_{k \in \mathcal{K}} P_{k}=P
\end{gathered}
$$

where "s.t." stands for "subject to", $\bar{P}_{e}$ denotes the system's 248 average BER and $\bar{P}_{e}^{(k)}$ denotes the average BER of user $k$. In 249 (3), $\cup\left\{\mathcal{F}_{k}, P_{k}\right\}$ stands for searching all the possible candidates 250 for all users, while $\cup\left\{\mathcal{F}_{k}, P_{k}\right\}^{*}$ contain the final results for 251 power- and subcarrier-allocation of all the users. In practice, 252 however, it is often very hard to solve the optimization problem 253 of (3). Since the average BER $\bar{P}_{e}$ in various of multicarrier 254 communications is usually dominated by the subcarrier with the 255 lowest SNR [8]. Consequently, in some references, such as in 256 [7], [19], [20], power- and subcarrier-allocation algorithms are 257 designed to maximize the minimum SNR of users.

According to [7], [8], power- and subcarrier-allocation can 259 be carried out separately without loss of much performance 260 but having much lower implementation complexity. Therefore, 261 in this contribution, we assume that power- and subcarrier- 262 allocation are executed separately in two steps. Specifically, 263 after subcarrier-allocation, power-allocation is carried out 264 according to the channels of the subcarriers allocated to dif- 265 ferent users. In this paper, the channel-inverse assisted power- 266 allocation is employed, which has been proved to be optimum 267 in the sense of maximizing the reliability. Under this power- 268 allocation strategy, user $k$ is allocated the power [8]

$$
P_{k}=P\left(\sum_{l=1}^{K} A_{l}^{-1}\right)^{-1} A_{k}^{-1}, \quad k \in \mathcal{K}
$$

where $A_{k}$ denotes the channel quality of the subcarrier assigned 270 to user $k$. After the power-allocation, it can be shown that the 271 SNR of user $k$ is

$$
\gamma_{k}=\gamma_{c}=P\left(\sum_{l \in \mathcal{K}} A_{l}^{-1}\right)^{-1}, \quad k \in \mathcal{K}
$$

which is independent of the index $k$, implying that all the users 273 attain the same SNR $\gamma_{c}$ and, hence, they also have the same 274 error probability.

From (5) we can know that, in order to maximize the SNR, 276 the subcarrier-allocation algorithms should be designed aiming 277 to maximize $\left(\sum_{l \in \mathcal{K}} A_{l}^{-1}\right)^{-1}$, yielding the optimization problem 278

$$
\begin{aligned}
\cup\left\{\mathcal{F}_{j}\right\}^{*} & =\arg \max _{\cup\left\{\mathcal{F}_{j}\right\}}\left\{\left(\sum_{l \in \mathcal{K}} A_{l}^{-1}\right)^{-1}\right\}, \\
\text { s.t. } \quad \cup_{j \in \mathcal{M}} \mathcal{F}_{j} & =\mathcal{K}, \quad \mathcal{F}_{j} \cap \mathcal{F}_{l}=\emptyset \text { for } j \neq l .
\end{aligned}
$$

To solve the above optimization problem, exhaustive search 279 may be carried out, which however has extremely high com- 280 plexity and prevents the algorithm from practical implementa- 281 tion, when the number of subcarriers and the number of users 282 are relatively high. In literature, the Hungarian algorithm [11] 283 is aimed to solve the optimization problem of (6) with lower 284 complexity than the exhaustive search. However, its complexity 285 is still too high for practical implementation, especially, when 286 there are a large number of subcarriers supporting many users, 287 which is usually the case in LTE/LTE-A systems. 288

In order to minimize the complexity, in this contribution, 289 we focus on the sub-optimum algorithms, which motivate to 290 
TABLE I

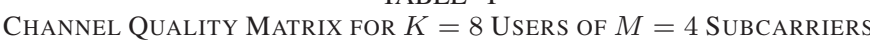

\begin{tabular}{|c||c|c|c|c|c|c|c|c|}
\hline S U & 0 & 1 & 2 & 3 & 4 & 5 & 6 & 7 \\
\hline \hline 0 & 3.73 & 4.95 & 5.06 & 0.34 & 2.37 & 5.04 & 1.59 & 3.42 \\
\hline 1 & 1.39 & 2.01 & 0.52 & 4.71 & 5.02 & 8.32 & 10.60 & 2.12 \\
\hline 2 & 0.41 & 1.63 & 4.52 & 0.87 & 0.91 & 3.50 & 2.49 & 0.65 \\
\hline 3 & 2.13 & 5.07 & 4.57 & 2.55 & 3.22 & 0.49 & 1.20 & 0.02 \\
\hline
\end{tabular}

291 maximize the SNR by maximizing the worst channel quality 292 of the subcarriers allocated to the users, as suggested by the 293 study in [7]. This is because, according to (6), the value 294 of $\left(\sum_{l \in \mathcal{K}} A_{l}^{-1}\right)^{-1}$ is mainly determined by the minimum of $295\left\{A_{0}, A_{1}, \ldots, A_{K-1}\right\}$. Correspondingly, the optimization prob296 lem can be stated as

$$
\begin{aligned}
\cup\left\{\mathcal{F}_{j}\right\}^{*} & =\arg \max _{\cup\left\{\mathcal{F}_{j}\right\}}\left\{\min _{l \in \mathcal{K}}\left\{A_{l}\right\}\right\}, \\
\text { s.t. } \quad \cup_{j \in \mathcal{M}} \mathcal{F}_{j} & =\mathcal{K}, \mathcal{F}_{j} \cap \mathcal{F}_{l}=\emptyset \text { for } j \neq l .
\end{aligned}
$$

297 Note that, the WSA algorithm in [7] has been designed to 298 solve the optimization problem of (7) for the downlink OFDMA 299 system. As our studies and performance results show, our 300 proposed subcarrier-allocation algorithms, including the WCA, $301 \mathrm{WCF}$, IWE-WCA as well as the IWE-WCF algorithms, are 302 capable of finding better solutions for subcarrier-allocation and 303 achieving better error performance than the WSA algorithm.

304 Note additionally that, in principle, the subcarrier-allocation 305 algorithms proposed in this paper as well as the WSA algorithm 306 [7] all belong to the greedy family, which motivate to attain high 307 throughput. Our algorithms can maintain all the merits of the 308 conventional greedy algorithm [5], while circumventing its dis309 advantage of low reliability. This is because our algorithms aim 310 to maximize the reliability via maximizing the achievable SNR. 311 Therefore, they do not generate a trade-off on the throughput, 312 since throughput is an increasing function of SNR.

\section{SubCARrier-Allocation Algorithms}

314 In this section, we first review the principles of two represen315 tative low-complexity subcarrier-allocation algorithms, namely 316 the greedy algorithm and the WSA algorithm. Their advan317 tages and drawbacks are analyzed, against which a range of 318 subcarrier-allocation algorithms are proposed and investigated. 319 Along with our analysis, an example is introduced, which 320 employs $M=4$ subcarriers to support $K=8$ mobile users. 321 Therefore, each subcarrier can be assigned to two users, which 322 are distinguished by their DS spreading codes of length $N=2$. 323 In this example, the channel qualities corresponding to the four 324 subcarriers of the eight users are illustrated in Table I, where the 325 first row and first column denote the user indexes and subcarrier 326 indexes, respectively. Furthermore, the total transmission power $327 P=1$ is assumed for the example considered. From the above 328 discussion, we can realize that the main difference between 329 the subcarrier-allocation in OFDMA systems and that in MC 330 DS-CDMA systems is that one subcarrier is only assigned to 331 one user in the OFDMA systems, while one subcarrier may be 332 assigned to multiple users in the MC DS-CDMA systems. Let 333 us first consider the greedy algorithm.

\section{A. Greedy Algorithm}

In the context of the greedy algorithm [5], a subcarrier 335 is always allocated to the two users (in contrast to one in 336 OFDMA) having the best channel qualities among the users 337 still requiring subcarriers. For the example considered, the 338 subcarrier-allocation is carried out one by one from the first 339 subcarrier to the last. Specifically, subcarrier 0 is allocated to 340 users 2 and 5, as they have the two highest channel qualities on 341 subcarrier 0 among the eight users. Hence, the allocation set for 342 subcarrier 0 is updated to $\mathcal{F}_{0}=\{2,5\}$. Similarly, subcarrier 1 is 343 allocated to users 4 and 6 , as they have the best channel qualities 344 among the remaining users for this subcarrier, yielding $\mathcal{F}_{1}=345$ $\{4,6\}$. Similarly, we can obtain $\mathcal{F}_{2}=\{1,3\}$ and $\mathcal{F}_{3}=\{0,7\}$. 346 According to the allocation results and (5), it can be shown that 347 the attainable SNR is given by $\gamma_{c}=\left(\sum_{k \in \mathcal{F}_{j}} A_{k}^{-1}\right)^{-1}=0.019,348$ while the worst (minimum) channel quality of the allocated 349 subcarriers is $\min _{k \in\left\{\mathcal{F}_{j}\right\}}\left\{A_{k, j}\right\}=0.02$, which dominates the 350 attainable SNR and hence the achievable error performance. 351

Explicitly, the greedy algorithm has the advantage of low- 352 complexity. However, at the later stages of allocation, the 353 algorithm may have to assign users the subcarriers with very 354 poor channel qualities, as there are no other options. As the 355 above example shows, at the last stage, subcarrier 3 has to be 356 allocated to user 7 , which results in the poorest channel quality 357 of $A_{7,3}=0.02$.

\section{B. Worst Subcarrier Avoiding Algorithm}

The WSA algorithm is designed to avoid assigning users the 360 subcarriers having the worst channel qualities [7]. With the aid 361 of the example of Table I, the principles of the WSA algorithm 362 can be illustrated as follows.

Firstly, for each of the subcarriers, the worst channel quality 364 is identified, denoted by bold font in (8). It can be readily known 365 that the worst channel qualities corresponding to the four sub- 366 carriers are $A_{0}^{(\min )}=0.34$ for subcarrier $0, A_{1}^{(\min )}=0.52$ for 367 subcarrier $1, A_{2}^{(\min )}=0.41$ for subcarrier 2 and $A_{3}^{(\min )}=0.02368$ for subcarrier 3. Secondly, the subcarriers are arranged in the 369 ascending order as $\{3,0,2,1\}$ according to their worst channel 370 qualities, forming a matrix shown as

371

$\left[\begin{array}{ccccccccc} & \mathrm{U} 0 & \mathrm{U} 1 & \mathrm{U} 2 & \mathrm{U} 3 & \mathrm{U} 4 & \mathrm{U} 5 & \mathrm{U} 6 & \mathrm{U} 7 \\ \mathrm{~S} 3 & 2.13 & \underline{5.07} & \underline{4.57} & 2.55 & 3.22 & 0.49 & 1.20 & \mathbf{0 . 0 2} \\ \mathrm{S} 0 & \underline{3.73} & 4.95 & 5.06 & \mathbf{0 . 3 4} & 2.37 & \underline{5.04} & 1.59 & 3.42 \\ \mathrm{~S} 2 & \mathbf{0 . 4 1} & 1.63 & 4.52 & 0.87 & \underline{0.91} & \underline{3.50} & \underline{2.49} & 0.65 \\ \mathrm{~S} 1 & 1.39 & 2.01 & \mathbf{0 . 5 2} & \underline{4.71} & \underline{5.02} & 8.32 & \underline{10.60} & \underline{2.12}\end{array}\right]$

where, again, the worst channel qualities are represented by 372 boldface values. Finally, based on the above-derived matrix, 373 the subcarriers are allocated to the eight users in the principles 374 of the greedy algorithm, as discussed in Section IV-A, from 375 the first row to the last row, yielding the allocation results 376 $\mathcal{F}_{0}=\{0,5\}, \mathcal{F}_{1}=\{3,7\}, \mathcal{F}_{2}=\{4,6\}$, and $\mathcal{F}_{3}=\{1,2\}$, cor- 377 responding to the underlined numbers in (8). With the aid of (5), 378 the attainable SNR is evaluated to be $\gamma_{c}=\left(\sum_{k \in \mathcal{F}_{j}} A_{k}\right)^{-1}=379$ 0.29 , when assuming the total transmission power $P=1.380$ Furthermore, from (8) we can know that the worst channel 381 
382 quality of the allocated subcarriers is $\min _{k \in\left\{\mathcal{F}_{j}\right\}}\left\{A_{k, j}\right\}=0.91$. 383 Explicitly, the WSA algorithm significantly improves both the 384 worst channel quality and the attainable SNR per subcarrier, in 385 comparison with that obtained by the greedy algorithm. Owing 386 to the above, the WSA algorithm is expected to achieve better 387 error performance than the greedy algorithm [7].

\section{C. Worst Case Avoiding Algorithm}

389 From the analysis in Section IV-B, we may classify the 390 WSA algorithm as a subcarrier-oriented WSA algorithm, which 391 is capable of avoiding assigning the $(M-1)$ worst channels 392 when there are in total $M$ subcarriers [7]. Specifically, for the 393 considered example, the WSA algorithm can guarantee not to 394 assign the three worst channels and, in most cases, the four 395 worst can be avoided. In the MC DS-CDMA systems where 396 the number of users is more than the number of subcarriers, 397 in order to achieve better error performance, the subcarrier398 allocation may be operated in the user-oriented mode, which 399 may avoid assigning more of the worst channels. Inspired by 400 the observation, in this subsection, we generalize the WSA 401 algorithm to a so-called worst case avoiding (WCA) algorithm, 402 the principles of which is first illustrated below.

403 When the WCA algorithm is employed, it always tries to 404 avoid as many as possible the worst channels. The WCA 405 algorithm is operated either in the subcarrier-oriented mode, 406 i.e., WSA, or in the user-oriented mode. Specifically, for the 407 example considered, as the number of users is higher than the 408 number of subcarriers, the user-oriented mode will avoid a 409 higher number of worst channels than the subcarrier-oriented 410 WSA algorithm. In this case, the WCA algorithm first arranges 411 the users in an ascending order of $\{7,3,0,5,2,4,6,1\}$ according 412 to their worst channel qualities of four subcarriers, yielding

$\left[\begin{array}{ccccccccc} & \mathrm{U} 7 & \mathrm{U} 3 & \mathrm{U} 0 & \mathrm{U} 5 & \mathrm{U} 2 & \mathrm{U} 4 & \mathrm{U} 6 & \mathrm{U} 1 \\ \mathrm{~S} 0 & \underline{3.42} & \mathbf{0 . 3 4} & \underline{3.73} & 5.04 & 5.06 & 2.37 & 1.59 & 4.95 \\ \mathrm{~S} 1 & 2.12 & \underline{4.71} & 1.39 & \underline{8.32} & \mathbf{0 . 5 2} & 5.02 & 10.60 & 2.01 \\ \mathrm{~S} 2 & 0.65 & 0.87 & \mathbf{0 . 4 1} & 3.50 & 4.52 & \mathbf{0 . 9 1} & \underline{2.49} & \underline{\mathbf{1 . 6 3}} \\ \mathrm{S} 3 & \mathbf{0 . 0 2} & 2.55 & 2.13 & \mathbf{0 . 4 9} & \underline{4.57} & \underline{3.22} & \mathbf{1 . 2 0} & 5.07\end{array}\right]$

413 In (9) the channel qualities in boldface are the worst channel 414 qualities of the users. Then, based on the ordered matrix (9), 415 the subcarrier-allocation is carried out based on the greedy 416 algorithm, one user at a stage, from the first to the last 417 column. Consequently, the allocation results are $\mathcal{F}_{0}=\{0,7\}$, $418 \mathcal{F}_{1}=\{3,5\}, \mathcal{F}_{2}=\{1,6\}$, and $\mathcal{F}_{3}=\{2,4\}$. It can be shown 419 that the SNR achieved by the WCA algorithm is $\gamma_{c}=0.41$, 420 and the worst channel quality of the allocated subcarriers is $421 \min _{k \in\left\{\mathcal{F}_{j}\right\}}\left\{A_{k, j}\right\}=1.63$.

422 Straightforwardly, the proposed WCA algorithm is capable 423 of achieving better allocation results than the WSA algorithm, 424 as the WSA is a special case of the WCA. For the considered 425 example, both the worst channel quality and the achievable 426 SNR are improved in comparison with that obtained by the 427 WSA algorithm. Furthermore, it can be shown that the WCA 428 algorithm is capable of preventing allocating at least $\max \{K-$ $429 N, M-1\}$ worst channels, instead of at least $(M-1)$ of the 430 WSA algorithm.
In summary, the WCA algorithm can be stated as follows.

Algorithm 1: (Worst Case Avoiding Algorithm)

Initialization

433

Subcarrier-oriented mode is chosen when $M \geq K$, other- 434 wise, user-oriented mode is selected when $M<K$. Set 435 $\tilde{\mathcal{M}}=\mathcal{M}, \tilde{\mathcal{K}}=\mathcal{K}$.

436

1) Worst channel quality identification

437

User-oriented mode-Find each user's worst channel 438 quality: $A_{k}^{(\min )}=\min _{j \in \mathcal{M}}\left\{A_{k, j}\right\}$.

439

Subcarrier-oriented mode-Find each subcarrier's worst 440 channel quality: $A_{j}^{(\min )}=\min _{k \in \mathcal{K}}\left\{A_{k, j}\right\} . \quad 441$

2) User (or Subcarrier) ordering 442

User-oriented mode-Arrange users in ascending order 443 according to the worst channel qualities as $\left\{i_{0}, i_{1}, \ldots, 444\right.$ $\left.i_{K-1}\right\}$, if $A_{i_{0}}^{(\mathrm{min})} \leq A_{i_{1}}^{(\mathrm{min})} \leq \cdots \leq A_{i_{K-1}}^{(\mathrm{min})} . \quad 445$ Subcarrier-oriented mode-Arrange subcarriers in as- 446 cending order according to the worst channel qualities as 447 $\left\{q_{0}, q_{1}, \ldots, q_{M-1}\right\}$, if $A_{q_{0}}^{(\min )} \leq A_{q_{1}}^{(\min )} \leq \cdots \leq A_{q_{M-1}}^{(\min )} .448$

3) Allocation

Based on the above-derived order, subcarrier-allocation is 450 carried out one-by-one:

451

User-oriented mode-First, at the $i_{k}$ th stage, subcarrier 452 $j^{*}$ is allocated to user $i_{k}: j^{*}=\arg \max _{j \in \tilde{\mathcal{M}}}\left\{A_{i_{k}, j}\right\}, i_{k} \in 453$ $\mathcal{K}$. Then, if subcarrier $j^{*}$ has been assigned to $N=K / M 454$ users, it is removed from $\tilde{\mathcal{M}}: \tilde{\mathcal{M}} \leftarrow \tilde{\mathcal{M}}-\left\{j^{*}\right\}$. $\quad 455$ Subcarrier-oriented mode - First, at the $q_{m}$ th stage, user 456 $k^{*}$ is allocated to subcarrier $q_{m}: k^{*}=\arg \max _{k \in \tilde{\mathcal{K}}}\left\{A_{k, q_{m}}\right\}, 457$ $q_{m} \in \mathcal{M}$. Then, if user $k^{*}$ has been assigned the required 458 number of subcarriers, it is deleted from $\tilde{\mathcal{K}}: \tilde{\mathcal{K}} \leftarrow \tilde{\mathcal{K}}-459$ $\left\{k^{*}\right\}$.

460

\section{Worst Case First Algorithm}

According to the WCA algorithm described in Section IV-C, 462 as the example shows, user 2 is allocated the subcarrier at the 463 fifth stage, as its worst channel quality is $A_{2,1}=0.52$, which 464 is the fifth worst of the users. However, from (9) we observe 465 that subcarriers 0 and 1 cannot be the options for user 2, as 466 each of these two subcarriers has been assigned to two users. 467 In this case, the worst channel quality of user 2's available 468 subcarriers becomes $A_{2,2}=4.52$, which is much larger than 469 that of users 4, 6, and 1's available subcarriers (which are 0.91, 470 1.2 , and 1.63 , respectively). Therefore, in order to maximize 471 the system's reliability, it would be beneficial to allocate the 472 subcarriers to users 4,6 , and 1 before assigning the subcarrier 473 to user 2.

Based on the above observation, we propose the WCF al- 475 gorithm, which re-order the users (or subcarriers) according to 476 the worst channel qualities of the available subcarriers (users). 477 Specifically for the MC DS-CDMA with $K>M$, during each 478 stage, the algorithm first finds the worst channel quality of 479 the unassigned users among only the subcarriers available for 480 allocation, rather than finding the worst channel quality of the 481 unsigned users among all the subcarriers, as done by the WCA 482 algorithm. In detail, for the example considered, the WCF 483 
484 algorithm completes the allocation user by user in 8 stages, 485 which can be demonstrated as

$\left[\begin{array}{ccccccccc} & \mathrm{U} 7 & \mathrm{U} 3 & \mathrm{U} 0 & \mathrm{U} 5 & \mathrm{U} 4 & \mathrm{U} 6 & \mathrm{U} 1 & \mathrm{U} 2 \\ \mathrm{~S} 0 & \underline{3.42} & \mathbf{0 . 3 4} & \underline{3.73} & 5.04 & 2.37 & 1.59 & 4.95 & 5.06 \\ \mathrm{~S} 1 & 2.12 & \underline{4.71} & 1.39 & \underline{8.32} & 5.02 & 10.60 & 2.01 & 0.52 \\ \mathrm{~S} 2 & 0.65 & 0.87 & \mathbf{0 . 4 1} & 3.50 & \mathbf{0 . 9 1} & \underline{2.49} & \mathbf{1 . 6 3} & \underline{\mathbf{4 . 5 2}} \\ \text { S3 } & \mathbf{0 . 0 2} & 2.55 & 2.13 & \mathbf{0 . 4 9} & \underline{3.22} & \underline{\mathbf{1 . 2 0}} & \underline{5.07} & 4.57\end{array}\right]$

486 where the eight columns stand for the eight stages of allocation, 487 the channel qualities in boldface are the minimum of the users' 488 channel qualities of the available subcarriers at the eight stages. 489 As shown in (10), at the first stage, the eight users' worst 490 channel qualities of the subcarriers are the same as those in 491 boldface in (9). In this case, user $7\left(A_{7,3}=0.02\right)$ is the worst 492 and it is first assigned subcarrier 0 with the best channel 493 quality of 3.42 among the four subcarriers. Similarly, as seen 494 in (10), users 3, 0, and 5 are assigned subcarriers 1, 0, and 1, 495 respectively, during the second, third and fourth stages. At this 496 moment, we can see from (10) that the worst channel qualities 497 of the available subcarriers for the four remaining users are $498 A_{1,2}=1.63$ for user $1, A_{2,2}=4.52$ for user $2, A_{4,2}=0.91$ for 499 user 4 and $A_{6,3}=1.20$ for user 6 , respectively. As we can see, 500 the worst channel quality of the subcarriers available to user 2 501 becomes $A_{2,2}=4.52$ instead of $A_{2,1}=0.52$, as subcarrier 1 502 (also subcarrier 0 ) has already been assigned to two users in 503 the previous four stages and cannot be assigned to other users. 504 Therefore, at the fifth stage, a subcarrier is assigned to user 4, 505 which is subcarrier 3. Similarly, subcarriers can be assigned 506 to users 6, 1, and 2. From (10) we can know that the final 507 allocation results are $\mathcal{F}_{0}=\{0,7\}, \mathcal{F}_{1}=\{3,5\}, \mathcal{F}_{2}=\{2,6\}$, 508 and $\mathcal{F}_{3}=\{1,4\}$. The achievable SNR of the system is $\gamma_{c}=$ 5090.49 and the worst channel quality of the assigned subcarriers 510 is $\min _{k \in\left\{\mathcal{F}_{j}\right\}}\left\{A_{k, j}\right\}=2.49$.

511 In comparison with the WCA algorithm, as shown in 512 Section IV-C, user 1 is forced to select subcarrier 2 at the last 513 stage, which results in the poorest channel quality of $A_{1,2}=1.63$. 514 By contrast, under the WCF algorithm, user 1 has two options 515 to choose either subcarrier 2 or subcarrier 3 at the seventh stage, 516 and is then assigned the better subcarrier 3, which results in a 517 channel quality of $A_{1,3}=5.07$, which is significantly higher 518 than $A_{1,2}=1.63$ obtained by the WCA algorithm.

519 When comparing the WCF the WCA, it is not hard to know 520 that the WCF algorithm is capable of yielding the highest 521 achievable SNR as well as the highest worst channel quality, 522 as demonstrated by the above example. As the above example 523 shows, the WCF algorithm successfully avoids assigning the 524 worst channel quality by preventing the unreasonable allocation 525 for user 2 at the fifth stage by the WCA algorithm. Therefore, 526 the proposed WCF algorithm provides a more reliable and 527 efficient way of subcarrier-allocation, while simultaneously 528 captures all the advantages of the WCA algorithm. In summary, 529 the WCF algorithm is stated as:

\section{Algorithm 2: (Worst Case First Algorithm)} Initialization

User-oriented mode is chosen when $M<K$, subcarrieroriented mode is used when $M \geq K$. Set $\tilde{\mathcal{K}}=\mathcal{K}, \tilde{\mathcal{M}}=\mathcal{M}$. Set $\mathcal{F}_{j}=\emptyset$ for all $j \in \mathcal{M}$.
Repeat

535

1) User-oriented mode-Identify the worst channel quality 536 of each user: $A_{k}^{(\min )}=\min _{j \in \tilde{\mathcal{M}}}\left\{A_{k, j}\right\}$, for all $k \in \tilde{\mathcal{K}} . \quad 537$ Subcarrier-oriented mode-Identify the worst channel 538 quality of each subcarrier: $A_{j}^{(\min )}=\min _{k \in \tilde{\mathcal{K}}}\left\{A_{k, j}\right\}$, for 539 all $j \in \tilde{\mathcal{M}}$.

540

2) User-oriented mode-Find the user with the minimum of 541 the worst channel qualities: $k^{*}=\arg \min _{k \in \tilde{\mathcal{K}}}\left\{A_{k}^{(\min )}\right\} . \quad 542$ Subcarrier-oriented mode-Find the subcarrier with the 543 minimum of the worst channel qualities: $j^{*}=544$ $\arg \min _{j \in \tilde{\mathcal{M}}}\left\{A_{j}^{(\min )}\right\}$.

545

3) User-oriented mode-Assign user $k^{*}$ the subcarrier with 546 the best channel quality: $q^{\prime}=\arg \max _{q \in \tilde{\mathcal{M}}}\left\{A_{k^{*}, q}\right\}$, then 547 $\mathcal{F}_{q^{\prime}} \leftarrow \mathcal{F}_{q^{\prime}} \bigcup\left\{k^{*}\right\}$.

548

Subcarrier-oriented mode-Allocate subcarrier $j^{*}$ to the 549 user with the best channel quality: $i^{\prime}=\arg \max _{i \in \tilde{\mathcal{K}}}\left\{A_{i, j^{*}}\right\}, 550$ then $\mathcal{F}_{j^{*}} \leftarrow \mathcal{F}_{j^{*}} \bigcup\left\{i^{\prime}\right\}$.

4) User-oriented mode-Remove user $k^{*}$ from $\tilde{\mathcal{K}}: \tilde{\mathcal{K}} \leftarrow \tilde{\mathcal{K}}-552$ $\left\{k^{*}\right\}$. Remove subcarrier $q^{\prime}$ from $\tilde{\mathcal{M}}$ if $\left|\mathcal{F}_{q^{\prime}}\right|=N: \tilde{\mathcal{M}} \leftarrow 553$ $\tilde{\mathcal{M}}-\left\{q^{\prime}\right\}$.

554

Subcarrier-oriented mode-Remove subcarrier $j^{*}$ from 555 $\tilde{\mathcal{M}}: \tilde{\mathcal{M}} \leftarrow \tilde{\mathcal{M}}-\left\{j^{*}\right\}$. Remove user $i^{\prime}$ from $\tilde{\mathcal{K}}$ if it has been 556 assigned the required number of subcarriers: $\tilde{\mathcal{K}} \leftarrow \tilde{\mathcal{K}}-\left\{i^{\prime}\right\} .557$

5) Stop if $\tilde{\mathcal{K}}=\emptyset$, or $\tilde{\mathcal{M}}=\emptyset$. 558

\section{Iterative Worst Excluding Algorithms}

559

In this section, we propose a general algorithm called as 560 the iterative worst excluding (IWE), which can be employed 561 in associated with various of subcarrier-allocation algorithms, 562 such as those considered in the previous sections. With the aid 563 of the IWE algorithm, the error rate performance of subcarrier- 564 allocation algorithms may achieve further improvement. Let us 565 first illustrate the principles of the IWE algorithm.

566

\section{A. Iterative Worst Excluding Algorithm}

As the name suggests, the proposed IWE algorithm aims to 568 achieve an improved BER performance by iteratively updating 569 the associated channel quality matrix. During each iteration, 570 the IWE algorithm removes the worst channel qualities of the 571 candidate subcarriers or the candidate users, before carrying 572 out the subcarrier-allocation. After the subcarrier-allocation at 573 an iteration, the allocation results obtained are compared with 574 those obtained from the last iteration, in order to observe 575 whether any performance improvement is gained. If there is 576 performance gain, the algorithm continues to the next iteration. 577 Finally, the algorithm stops, when there is no further perfor- 578 mance improvement. In the followings, we demonstrate the 579 principles of the IWE algorithm in conjunction with the WCF 580 subcarrier-allocation algorithm, which can be referred to as the 581 IWE-WCF algorithm. Furthermore, we compare the IWE-WCF 582 algorithm with the other algorithms proposed in the previous 583 sections.

584

In the context of the IWE-WCF algorithm, the WCF al- 585 gorithm is first carried out based on the channel quality 586 matrix given in Table I during the first (initial) iteration. 587 


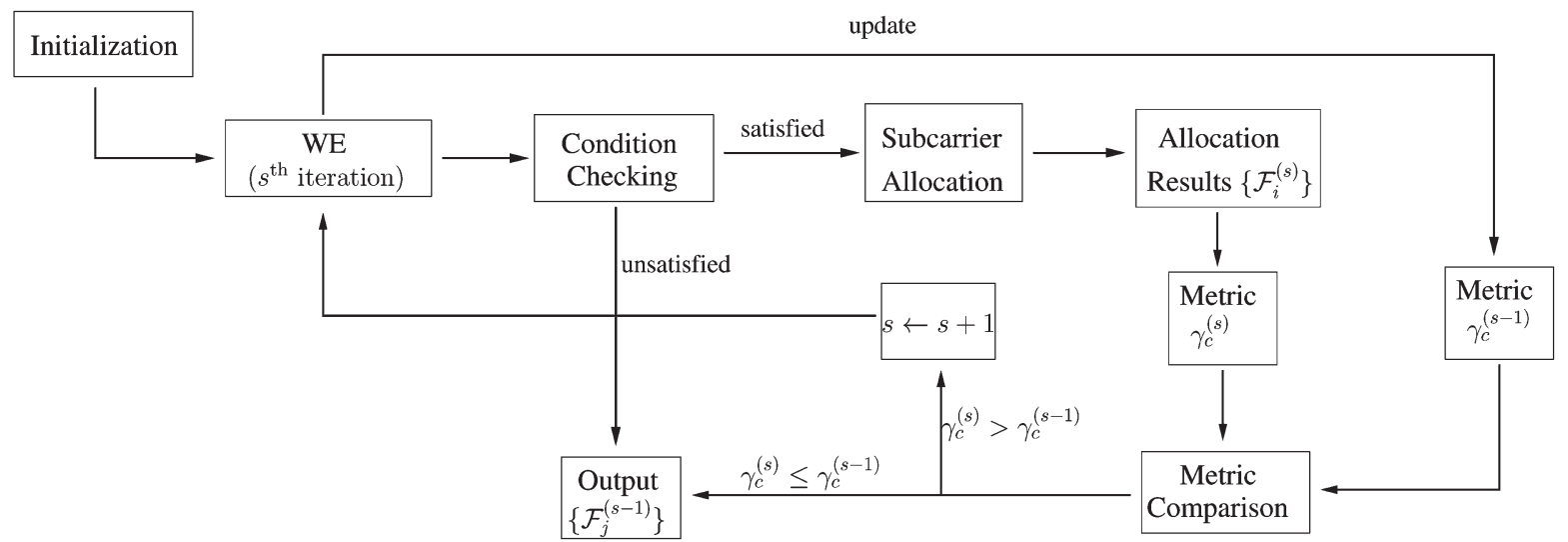

Fig. 1. Flowchart showing the steps of the IWE algorithm.

588 Correspondingly, the allocation results are given in the 589 Section IV-D and the attainable SNR is $\gamma_{c}^{(1)}=0.49$, where the 590 superscript of (1) indicates the first iteration. At the second 591 iteration, the worst channel qualities of the eight users are 592 eliminated before operating again the WCF algorithm, in order 593 to avoid assigning them to users. More specifically, the process 594 of the second iteration can be shown with the aid of (11)

$$
\left[\begin{array}{ccccccccc} 
& \mathrm{U} 7 & \mathrm{U} 3 & \mathrm{U} 0 & \mathrm{U} 6 & \mathrm{U} 4 & \mathrm{U} 5 & \mathrm{U} 2 & \mathrm{U} 1 \\
\mathrm{~S} 0 & 3.42 & \times & \underline{3.73} & \mathbf{1 . 5 9} & 2.37 & 5.04 & 5.06 & 4.95 \\
\mathrm{~S} 1 & 2.12 & \underline{4.71} & \mathbf{1 . 3 9} & \underline{10.60} & 5.02 & 8.32 & \times & 2.01 \\
\mathrm{~S} 2 & \mathbf{0 . 6 5} & \mathbf{0 . 8 7} & \times & 2.49 & \times & \underline{\mathbf{3 . 5 0}} & \underline{\mathbf{4 . 5 2}} & \times \\
\mathrm{S} 3 & \times & 2.55 & 2.13 & \times & \underline{\mathbf{3 . 2 2}} & \times & \underline{4.57} & \underline{\mathbf{5 . 0 7}}
\end{array}\right]
$$

595 where " $\times$ " stands for the worst channel quality of an user 596 which is removed before the subcarrier-allocation, referred to 597 as worst excluding (WE). After the WE, we can see in (11) that 598 subcarrier 0 can be allocated to any of the remaining 7 users. 599 We define these 7 users as the candidate users of subcarrier 0 , 600 expressed as $\tilde{\mathcal{F}}_{0}=\{0,1,2,4,5,6,7\}$. Simultaneously, we can 601 see that subcarrier 1 also has 7 candidate users. However, both 602 subcarrier 2 and 3 have only five candidate users.

603 Following the WE process, the algorithm carries out the 604 condition checking, in order to know whether the subcarrier605 allocation can be completed based on the updated channel 606 quality matrix. In order to fulfill the allocation, two conditions 607 have to be met. Otherwise, the following subcarrier-allocation 608 will not be carried out and the algorithm stops. In detail, the two 609 conditions are as follows.

610 Condition (a): The number of candidate users of each subcarrier 611 exceeds, $K / M$, of the number of users to be assigned to 612 one subcarrier. This condition can be expressed as

$$
\left|\tilde{\mathcal{F}}_{j}\right| \geq K / M, \quad \forall j \in \mathcal{M} \text {. }
$$

613 Condition (b): Each subcarrier can only be assigned to $K / M$ 614 different users and each user is only assigned one subcar615 rier, which can be expressed as

$$
\left|\tilde{\mathcal{F}}_{j} \cup \tilde{\mathcal{F}}_{q}\right| \geq 2 K / M, \quad j \neq q, \quad \forall j, q \in \mathcal{M} .
$$

Specifically, for the example considered, we can observe 616 from the updated matrix in (11) that the above two condi- 617 tions can be met. Thus, it guarantees that each subcarrier 618 can be allocated to two different users and each user attains 619 one subcarrier. Therefore, we can proceed the WCF algorithm 620 based on the updated matrix of (11). This process can also be 621 shown with the aid of (11), where the boldface value under 622 each user is the worst channel quality among the remaining 623 users. Upon following the principles of the WCF algorithm, 624 the new allocation results can be obtained, which are shown 625 by the underlined values in (11). The results are $\mathcal{F}_{0}^{(2)}=\{0,7\}, 626$ $\mathcal{F}_{1}^{(2)}=\{3,6\}, \mathcal{F}_{2}^{(2)}=\{2,5\}$, and $\mathcal{F}_{3}^{(2)}=\{1,4\}$. It can be 627 shown that the achievable SNR of the system is $\gamma_{c}^{(2)}=0.53,628$ while the worst channel quality of the allocated subcarriers is 629 $\min _{k \in\left\{\mathcal{F}_{j}^{(2)}\right\}}\left\{A_{k, j}\right\}=3.42$.

From the results of the second iteration, we can see that both 631 the SNR and the worst channel quality are improved in compar- 632 ison with those obtained from the first iteration. Therefore, the 633 IWE-WCF algorithm continues to the third iteration, and the 634 WE process is again first carried out, yielding

$$
\left[\begin{array}{ccccccccc} 
& \mathrm{U} 0 & \mathrm{U} 1 & \mathrm{U} 2 & \mathrm{U} 3 & \mathrm{U} 4 & \mathrm{U} 5 & \mathrm{U} 6 & \mathrm{U} 7 \\
\mathrm{~S} 0 & 3.73 & 4.95 & 5.06 & \times & \times & 5.04 & \times & 3.42 \\
\mathrm{~S} 1 & \times & \times & \times & 4.71 & 5.02 & 8.32 & 10.60 & 2.12 \\
\mathrm{~S} 2 & \times & \times & \times & \times & \times & \times & 2.49 & \times \\
\mathrm{S} 3 & 2.13 & 5.07 & 4.57 & 2.55 & 3.22 & \times & \times & \times
\end{array}\right]
$$

Then, the two required conditions are checked. Explicitly, the 636 candidate user set of subcarrier 2 contains only one user and 637 becomes $\tilde{\mathcal{F}}_{2}=\{6\}$. However, for the example considered, each 638 subcarrier is required to be allocated to $N=2$ users. Hence, 639 condition (a) described in (12) is not satisfied, and the algorithm 640 hence stops. Consequently, the results obtained from the second 641 iteration are taken as the final allocation results.

642

For convenience, the main steps of the IWE assisted 643 subcarrier-allocation algorithms can be described by the flow 644 chart in Fig. 1. In detail, during the initialization of the IWE 645 algorithm, with the specific subcarrier-allocation algorithm is 646 chosen, and the initial (first) iteration of subcarrier-allocation is 647 
648 carried out. After the initialization, the IWE scheme proceeds to 649 the second iteration, and sets $s=2$. During each iteration with $650 s \geq 2$, the WE process is first carried out, as shown in the figure. 651 Note that, the WE can be operated either in user direction or 652 in subcarrier direction, which is dependent on the subcarrier653 allocation algorithm employed, the number of subcarriers as 654 well as the number of users involved. For example, when the 655 IWE-WCF algorithm is employed, the WE is carried out in 656 user direction. By contrast, when the IWE-WSA algorithm is 657 used, the WE process is operated in subcarrier direction, i.e., 658 the worst channel quality of each of the subcarriers is removed. 659 As shown in Fig. 1, following the WE block, the algorithm 660 checks the conditions for assignment. When the two conditions 661 as mentioned in this section are satisfied, it proceeds to the 662 subcarrier-allocation. Otherwise, the IWE algorithm stops and 663 takes the results obtained in the $(s-1)$ th (previous) iteration as 664 the final subcarrier-allocation. If the sth iteration of subcarrier665 allocation is carried out, the allocation results of the sth (cur666 rent) iteration are compared with those of the previous iteration 667 against the performance metric. If performance is improved, the 668 algorithm continues to the next iteration. Otherwise, the IWE 669 algorithm stops and the allocation results from the previous 670 iteration are taken as the final allocation results.

\section{B. Characteristics of Iterative Worst Excluding Algorithm}

672 The IWE algorithm employs a range of advantages in the 673 sense of improving the error performance in comparison with 674 the various subcarrier-allocation algorithms found in refer675 ences. First, the IWE algorithm can be easily implemented in 676 conjunction with an existing subcarrier-allocation algorithm, in 677 order to enhance its performance, as discussed in Section V-A. 678 The core of the IWE algorithm is the WE process, which me679 liorates the channel quality matrix prior to operating subcarrier680 allocation. Based on the improved channel quality matrix, the 681 subcarrier-allocation followed can hence improve the error per682 formance. Second, the subcarrier-allocation algorithm assisted 683 by the IWE algorithm can always guarantee error performance 684 improvement in comparison with that without using the IWE. 685 In Section V-A, we only described the operation procedure 686 of the IWE-WCF algorithm. Similarly, we can also form the 687 IWE aided WSA (IWE-WSA) algorithm, the IWE aided WCA 688 (IWE-WCA) algorithm, etc., the performance of which will be 689 evaluated in Section VII. It should be noted that, the greedy 690 algorithm was designed not to maximize the minimum of 691 channel qualities as the optimization problem given in (7). 692 Hence, the IWE algorithm may not assist the greedy algorithm 693 and its extensions in improving the error performance. Finally, 694 from our studies, we find that the IWE algorithm is usually 695 operated with a low number of iterations, which guarantees the 696 IWE aided algorithms low complexity.

697 As the number of iterations required by the IWE algorithm is 698 an important factor, which affects the performance and com699 plexity of the associated subcarrier-allocation algorithms, in 700 Table II, we summarize the average number of iterations re701 quired by the various IWE aided subcarrier-allocation algo702 rithms for some cases. For this table, we assumed for the 703 considered downlink MC DS-CDMA system that all subcarri-

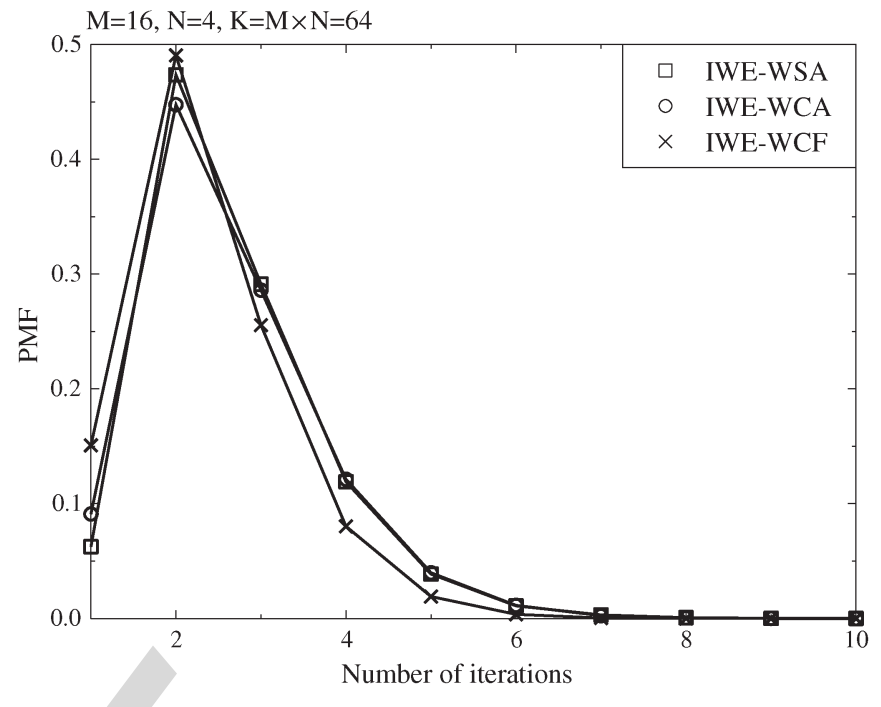

Fig. 2. Distribution of the number of iterations required by the IWE aided subcarrier-allocation algorithms.

ers of all users experience independent Rayleigh fading and the 704 Gaussian noise of the same variance. Furthermore, we assumed 705 that the number of users supported by the system is $K=M N .706$ Each of the results in the table was obtained by averaging 707 over the outcomes of $10^{5}$ simulations. From the results, we 708 can observe that the three IWE aided subcarrier-allocation 709 algorithms always require a low average number of iterations, 710 which is $\bar{S}<3$ for all the considered cases. Moreover, from the 711 table, a few other observations can be identified. First, given 712 a constant $N$ value, it can be shown that the average number 713 of iterations normalized by the number of subcarriers $M, 714$ i.e., $\bar{S} / M$, decreases explicitly as $M$ increases, even though, 715 for most cases, the average number of iterations $\bar{S}$ slightly 716 increases as $M$ becomes larger. Second, for most cases, $\bar{S}$ in 717 general becomes smaller as the spreading factor increases for a 718 constant $M$. Furthermore, the IWE-WSA algorithm requires in 719 average a slightly bigger number of iterations than the other two 720 algorithms considered. This is mainly because the IWE-WSA 721 algorithm carries out the WE operations in subcarrier direction, 722 while the other two algorithms run the WE operations in user 723 direction.

Furthermore, in Fig. 2, we illustrate the probability mass 725 function (PMF) of the number of iterations required by the three 726 IWE aided subcarrier-allocation algorithms, where the results 727 are obtained from $10^{5}$ realizations. Associated with the studies, 728 we assumed $M=16, K=64$, and $N=4$. It can be observed 729 that the number of iterations is a variable and, for most cases, 730 the allocation requires 2 iterations. However, the allocation 731 process sometimes requires up to 6 iterations. Furthermore, 732 the probability of requiring 8 iterations is nearly zero, which 733 is still much smaller than the number of users $K=64$. From 734 Table II and Fig. 2, we therefore can conclude that the IWE 735 aided algorithms usually demand a low number of iterations, 736 which ensures a low complexity for implementation. Note that, 737 in practice, we may set the maximum number of iterations to 738 three or four, which guarantees the most of the available gain, 739 while limit the complexity. 
TABLE II

Average Number of Iterations for the IWE Aided SubCARrier-Allocation Algorithms

\begin{tabular}{|c|c|c|c|c|c|c|c|c|c|c|c|c|}
\hline Algorithm & \multicolumn{4}{|c|}{ IWE-WCF } & \multicolumn{4}{|c|}{ IWE-WCA } & \multicolumn{4}{|c|}{ IWE-WSA } \\
\hline${ }_{N} M$ & 4 & 8 & 16 & 32 & 4 & 8 & 16 & 32 & 4 & 8 & 16 & 32 \\
\hline 1 & 1.76 & 2.14 & 2.44 & 2.58 & 2.20 & 2.75 & 2.74 & 2.73 & 2.20 & 2.75 & 2.74 & 2.73 \\
\hline 2 & 1.70 & 2.12 & 2.40 & 2.55 & 1.96 & 2.45 & 2.65 & 2.72 & 2.42 & 2.64 & 2.67 & 2.71 \\
\hline 4 & 1.60 & 2.03 & 2.34 & 2.52 & 1.78 & 2.30 & 2.61 & 2.71 & 2.43 & 2.55 & 2.65 & 2.70 \\
\hline 8 & 1.49 & 1.89 & 2.25 & 2.46 & 1.63 & 2.18 & 2.56 & 270 & 242 & 251 & 264 & 2.69 \\
\hline
\end{tabular}

741

742 In this section, we analyze the complexity of the proposed 743 subcarrier-allocation algorithms and that of the other related 744 algorithms. In our analysis, we assume that the same power745 allocation scheme is used for all the subcarrier-allocation al746 gorithms. Furthermore, the complexity reflects the number of 747 comparisons required by the subcarrier-allocation algorithms.

748 First, the complexity of the greedy algorithm and that of the 749 WSA algorithm can be found, for example, in [7], which are 750 both $\mathcal{O}\left(K^{2}\right)$ for the MC DS-CDMA systems with $K \geq M$. 751 Specifically, the number of comparisons required by the WSA 752 algorithm can be expressed as

$$
\mathcal{C}^{(\mathrm{WSA})}=M(K-1)+2 M \ln M+\frac{1}{2} K(K-1) .
$$

753 The complexity of the WCA algorithm depends on the 754 specific operations. First, the $K$ users are ordered from the 755 worst to the best according to their worst channel qualities. This 756 process requires $K(M-1)+2 K \ln K$ comparisons. Then, 757 for the subcarrier-allocation, the upper-bound happens when 758 each subcarrier is assigned to $(N-1)$ users during the first $759(K-M)$ stages. In this case, $(K-M)(M-1)+M(M-$ 760 1)/2 comparisons are required. When considering the above 761 analysis, the number of comparisons required by the WCA 762 algorithm satisfies

$$
\begin{aligned}
\mathcal{C}^{(\mathrm{WCA})} \leq & K(M-1)+2 K \ln K+(K-M)(M-1) \\
& +\frac{1}{2} M(M-1) \\
\leq & \left(2 K-\frac{M}{2}\right)(M-1)+2 K \ln K
\end{aligned}
$$

763 From (16), we can be implied that the WCA algorithm has a 764 complexity of $\mathcal{O}(K M)$.

765 Similarly, the complexity of the WCF algorithm has an 766 upper-bound, which happens when each of the $M$ subcarriers 767 is assigned to $(N-1)$ users during the first $(K-M)$ alloca768 tion stages. In this case, $K(M-1)$ comparisons are needed 769 for the $K$ users to find their worst channel qualities during 770 the first $(K-M+1)$ stages. Then, $\sum_{m=2}^{M-1}(M-m)=(M-$ $7711)(M-2) / 2$ comparisons are required for re-identifying the 772 worst channel quality during the last $(M-1)$ stages. More773 over, during each stage, the WCF algorithm finds the minimum 774 of the channel qualities of the $k(k=K, K-1, \ldots, 1)$ avail775 able users, which requires $K(K-1) / 2$ comparisons. Except 776 user ordering, the allocation process of the WCF algorithm is 777 the same as that of the WCA algorithm, which requires $(K-$ $778 M)(M-1)+M(M-1) / 2$ comparisons. Consequently, the upper-bound for the number of comparisons required by the 779 WCF algorithm can be expressed as

$$
\begin{aligned}
\mathcal{C}^{(\mathrm{WCF}) \leq} & K(M-1)+\frac{1}{2}(M-1)(M-2)+\frac{1}{2} K(K-1) \\
& +(K-M)(M-1)+\frac{1}{2} M(M-1) \\
\leq & (2 K-1)(M-1)+\frac{1}{2} K(K-1)
\end{aligned}
$$

According to (17), we can readily know that the WCF algorithm 781 has a complexity of $\mathcal{O}\left(K^{2}\right)$, as $K>M$ is assumed. 782

Let us now consider the complexity of the IWE-WSA algo- 783 rithm. First, during the $s$ th iteration, the WE process searches 784 for the worst channel qualities of the $M$ subcarriers, which have 785 already been identified by the WSA operations during the $(s-786$ 1 )th iteration. Therefore, there is no complexity contribution by 787 the WE process during the $s$ th iteration. Second, we can easily 788 find that the condition checking requires $\mathcal{C}^{\text {(checking) }}=M+789$ $M(M-1) / 2$ operations during the $s$ th $(s \geq 2)$ iteration. Note 790 that, at the $s$ th iteration, the number of comparisons required 791 by the WSA-assisted subcarrier-allocation is $\mathcal{C}^{\text {(allocation) }}(s)=792$ $\mathcal{C}^{\text {(WSA) }}-\mathcal{C}^{\text {(reduce) }}(s)$, where $\mathcal{C}^{\text {(reduce) }}(s)=2 M(s-1)$ de- 793 notes the number of comparisons reduced as a result that some 794 of the worst channels are removed by the WE process. When 795 considering all the above, the number of comparisons required 796 by the IWE-WSA algorithm can be expressed as

797

$$
\begin{aligned}
\mathcal{C}^{(\text {IWE-WSA })}= & (S-1) \mathcal{C}^{\text {(checking })}+\sum_{s=1}^{S} \mathcal{C}^{\text {(allocation })}(s) \\
= & \left(\frac{1}{2} S K+S M\right)(K-1) \\
& +\left(\frac{1}{2} M^{2}+\frac{1}{2} M-S M\right)(S-1)+2 S M \ln M
\end{aligned}
$$

when assuming that $S$ iterations are used. Equation (18) shows 798 a complexity of $\mathcal{O}\left(S K^{2}\right)$ for the IWE-WSA algorithm. 799

In the context of the IWE-WCA and IWE-WCF algorithms, 800 their complexity can be analyzed in the similar way as that 801 for the IWE-WSA algorithm, in conjunction with WCA and 802 WCF algorithms, respectively. It can be shown that the num- 803 ber of comparisons required by these two algorithms can be 804 expressed as

$$
\begin{aligned}
\mathcal{C}^{(\text {IWE-WCA })} & \leq\left(2 S K-\frac{1}{2} S M\right)(M-1) \\
+\left(\frac{1}{2} M^{2}\right. & \left.+\frac{1}{2} M-S K\right)(S-1)+2 S K \ln K,
\end{aligned}
$$


TABLE III

COMPLEXITY OF VARIOUS SUBCARRIER-ALLOCATION ALGORITHMS

\begin{tabular}{|c||c|}
\hline Algorithm & Complexity \\
\hline \hline Hungarian & $\mathcal{O}\left(K^{3}\right)[11]$ \\
\hline Greedy & $\mathcal{O}\left(K^{2}\right)$ \\
\hline WUF Greedy & $\mathcal{O}\left(K^{2}\right)$ \\
\hline Maximal Greedy & $\mathcal{O}\left(\alpha K^{2}\right)$ \\
\hline WSA & $\mathcal{O}\left(K^{2}\right)$ \\
\hline WCA & $\mathcal{O}(K M)$ \\
\hline WCF & $\mathcal{O}\left(K^{2}\right)$ \\
\hline IWE-WSA & $\mathcal{O}\left(S K^{2}\right)$ \\
\hline IWE-WCA & $\mathcal{O}\left(S K^{2}\right)$ \\
\hline IWE-WCF & $\mathcal{O}\left(S K^{2}\right)$ \\
\hline
\end{tabular}

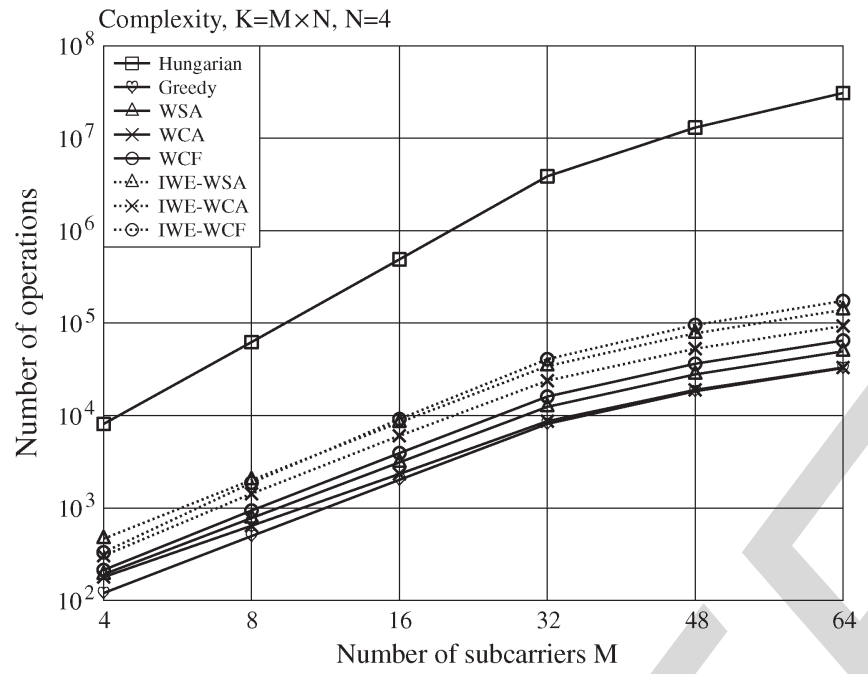

Fig. 3. Number of comparisons required by various subcarrier-allocation algorithms when $N=4$.

$$
\begin{aligned}
& \mathcal{C}^{(\mathrm{IWE}-\mathrm{WCF})} \leq \frac{1}{2} S K(K-1)+(2 S K-S)(M-1) \\
&+\left(\frac{1}{2} M^{2}+\frac{1}{2} M-S K\right)(S-1)
\end{aligned}
$$

806 respectively. Therefore, the complexity of both the IWE-WCA 807 and the IWE-WCF algorithms are $\mathcal{O}\left(S K^{2}\right)$.

808 In Table III, we summarize the complexity of the various 809 subcarrier-allocation algorithms. Note that, the maximal greedy 810 algorithm [10] requires a complexity of $\mathcal{O}\left(\alpha K^{2}\right)$, where $\alpha(\geq$ $811 M)$ is the size of the search space. In Section VII, we assume 812 that the maximal greedy algorithm uses a random search space 813 having the size $\alpha=M$. Furthermore, in Figs. 3 and 4, we 814 compare the number of operations required by the various 815 subcarrier-allocation algorithms with respect to the number of 816 subcarriers employed by the MC DS-CDMA systems. Note 817 that, in both figures, the number of operations are either the 818 exact values or the upper-bound of the algorithms. The number 819 of comparisons of the IWE algorithms were obtained from 820 (18)-(20). From both figures, we can see that the greedy and 821 WCA algorithms always require the least number of com822 parisons, while the Hungarian algorithm [11], [21] needs the 823 highest number of comparisons. When $N=4$ in Fig. 3, the 824 greedy algorithm demands the lowest number of comparisons 825 when $M \leq 32$. However, when $N=8$ in Fig. 4, the WCA 826 algorithm always has the lowest operations. Observing from the

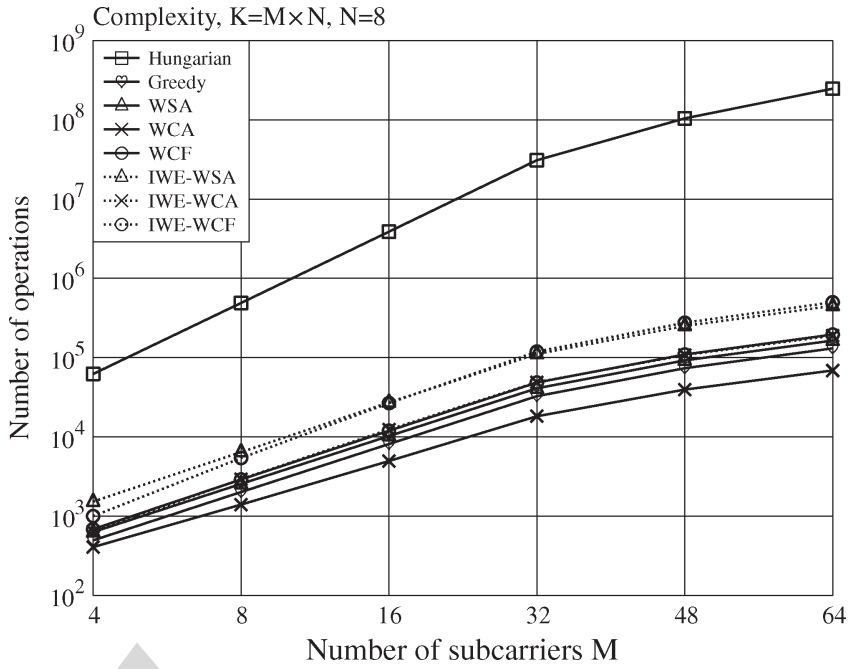

Fig. 4. Number of comparisons required by various subcarrier-allocation algorithms when $N=8$.

two figures, we can know that the complexity of the proposed 827 WCA and WCF algorithms are at the same level as that of 828 the WSA and greedy algorithm. Moreover, for the considered 829 examples, we find that the number of comparison required 830 by the IWE-aided subcarrier-allocation algorithms is slightly 831 less than twice of the number of comparisons required by the 832 original corresponding algorithms without invoking the IWE 833 algorithm.

\section{PERformance Results}

In this section, we provide a range of simulation results, in 836 order to demonstrate and compare the achievable error per- 837 formance of the downlink MC DS-CDMA systems employing 838 the proposed and the other subcarrier-allocation algorithms 839 considered. In our studies, we assume the Quadrature Phase- 840 Shift Keying (QPSK) baseband modulation and that all the 841 subcarriers experience independent flat Rayleigh fading. The 842 number of users supported by the MCDS-CDMA is $K=M N, 843$ with $M$ being the number of subcarriers and $N$ the length of 844 the orthogonal DS spreading codes. Furthermore, for all the 845 subcarrier-allocation algorithms considered, we assume that the 846 channel-inverse assisted power-allocation is employed, under 847 the constraint that the total transmission power is $P=K . \quad 848$

Fig. 5 demonstrates the BER performance of the MC 849 DS-CDMA system employing various of subcarrier-allocation 850 algorithms, when $K=64$ users are supported by $M=16851$ subcarriers. Hence, each subcarrier supports 4 users. From the 852 figure, we can obtain the following observations. First, the 853 Hungarian algorithm gives the best BER performance, while 854 the greedy algorithm yields the worst performance. Both the 855 WUF greedy algorithm [9] and the maximal greedy algorithm 856 [10], which assumes a random search space of size $\alpha=M, 857$ slightly outperform the greedy algorithm. As the greedy-class 858 algorithms aim to maximize the sum of the channel qualities, 859 rather than maximizing the reliability, such as the SNR in (5), 860 the greedy-class algorithms in general achieve poorer BER 861 performance than the other reliability motivated algorithm. 862 Second, as seen in Fig. 5, the proposed WCA, WCF, especially 863 


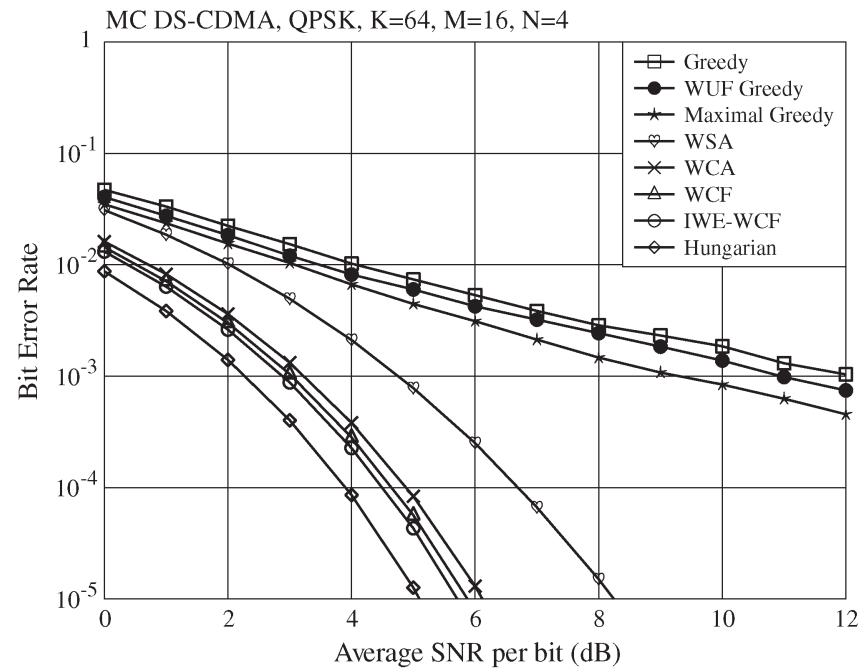

Fig. 5. BER comparison of the downlink MC DS-CDMA systems employing various subcarrier-allocation algorithms, when subcarriers experience independent Rayleigh fading.

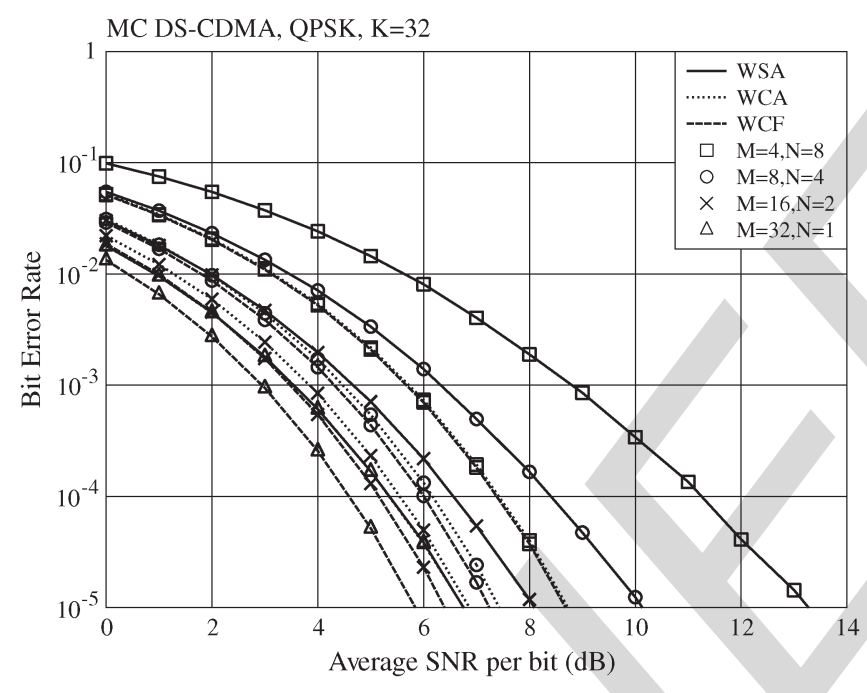

Fig. 6. BER comparison of the downlink MC DS-CDMA systems employing the WSA, WCA, and WCF algorithms, when subcarriers experience independent Rayleigh fading.

864 the IWE-WCF algorithms are capable of significantly out865 performing the greedy-class algorithms as well as the WSA 866 algorithm. Third, for the specific system parameters consid867 ered, the WCF algorithm has better BER performance than 868 the WCA algorithm. This is because the WCF algorithm can 869 avoid assignment of more number of worst subcarriers than the 870 WCA algorithm. Finally, by invoking the IWE scheme, further 871 error performance improvement can be attained with a penalty 872 of double complexity. The achievable BER of the IWE-WCF 873 algorithm is close to that achieved by the Hungarian algorithm, 874 and the difference is only $0.7 \mathrm{~dB}$.

875 Fig. 6 compares the BER performance of the MC DS-CDMA 876 systems employing the WSA, WCA and the WCF algorithms 877 for $K=32$ users. In general, the proposed WCA and WCF 878 algorithms always yield better BER performance than the WSA 879 algorithm. As discussed in Section IV, the WSA algorithm 880 implements the assignment by avoiding the worst channel 881 qualities in a subcarrier-oriented mode. Hence, its performance

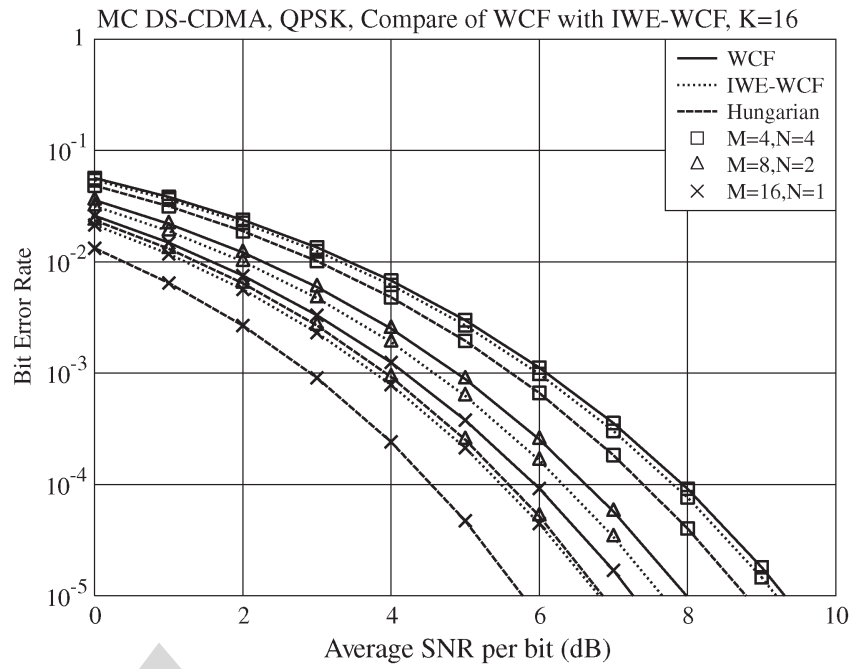

Fig. 7. BER comparison of the downlink MC DS-CDMA systems employing the WCF and the IWE-WCF algorithms, when subcarriers experience independent Rayleigh fading.

depends on the frequency-selective diversity. By contrast, for 882 the MC DS-CDMA systems employing DS spreading, the 883 number of users supported is usually higher than the number 884 of subcarriers, as considered in Fig. 6. In this case, the WCA 885 and WCF algorithms avoid the worst channel qualities in a user- 886 oriented mode and achieve much higher diversity than the WSA 887 scheme. Furthermore, from Fig. 6 we observe that, when given 888 $K=M N$ a constant, the BER performance of the three algo- 889 rithms improves as $M$ becomes larger. The reason behind the 890 observation is that we assumed that all subcarriers experience 891 independent fading regardless of the number of subcarriers. 892 This assumption implies that more subcarriers results in higher 893 diversity. In this case, the advantage of the WCA algorithm 894 over the WSA algorithm becomes smaller as the ratio of $K / M 895$ becomes bigger. Furthermore, when $M=K=32$ and $N=1,896$ both the WCA and WSA achieve the same BER, as, in this case, 897 the MC DS-CDMA is reduced to an OFDMA system without 898 T-domain spreading. Consequently, the user-oriented diversity 899 is the same as the subcarrier-oriented diversity. By contrast, as 900 shown in Fig. 6, the advantage of the WCF algorithm over the 901 WCA algorithm is enhanced as $M$ increases, when given $K=902$ $M N$ a constant. Specifically, when $M=32$ and $N=1$, the 903 WCF algorithm has $0.6 \mathrm{~dB}$ SNR gain over the WCA algorithm 904 at the BER of $10^{-5}$. From the above, we can know that, when 905 all subcarriers experience independent fading, the number of 906 subcarriers has a significant impact on the performance of the 907 considered subcarrier-allocation algorithms.

908

Figs. 7-9 show the BER gain of employing the IWE algo- 909 rithm for the WCF, WCA and WSA algorithms, respectively. 910 Under the various cases, the BER improvement can be obtained 911 by introducing the IWE algorithm. Thus, this observation con- 912 firms the benefit of using the IWE algorithm in association 913 with subcarrier-allocation algorithms. By comparing the three 914 figures, we observe that the IWE-WCF algorithm always has 915 the best BER performance, while the IWE-WSA has the worst 916 performance among the three IWE aided algorithms. This ob- 917 servation maintains the same for the three algorithms without 918 using the IWE algorithm in Fig. 6. From Figs. 7 and 8, we 919 


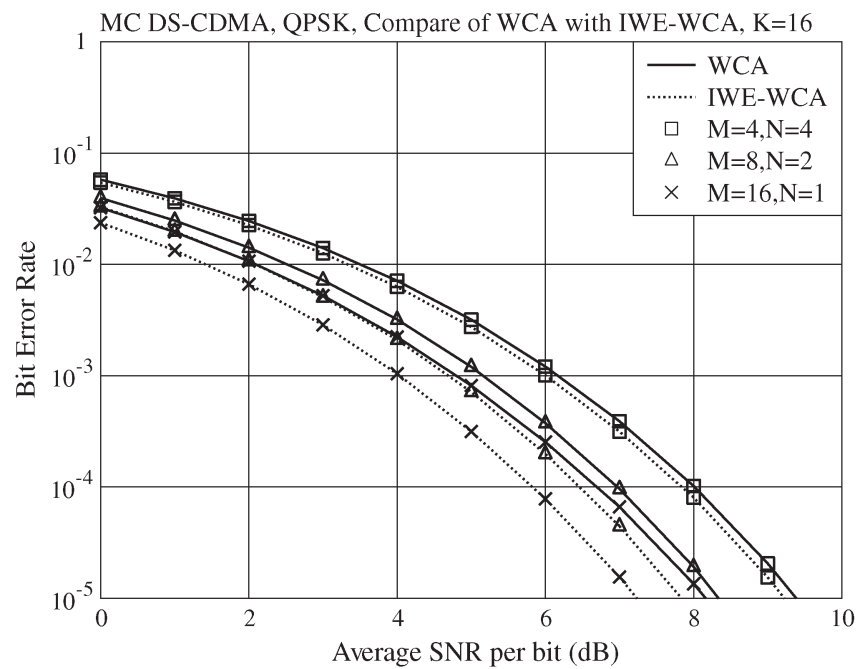

Fig. 8. BER comparison of the downlink MC DS-CDMA systems employing the WCA and the IWE-WCA algorithms, when subcarriers experience independent Rayleigh fading.

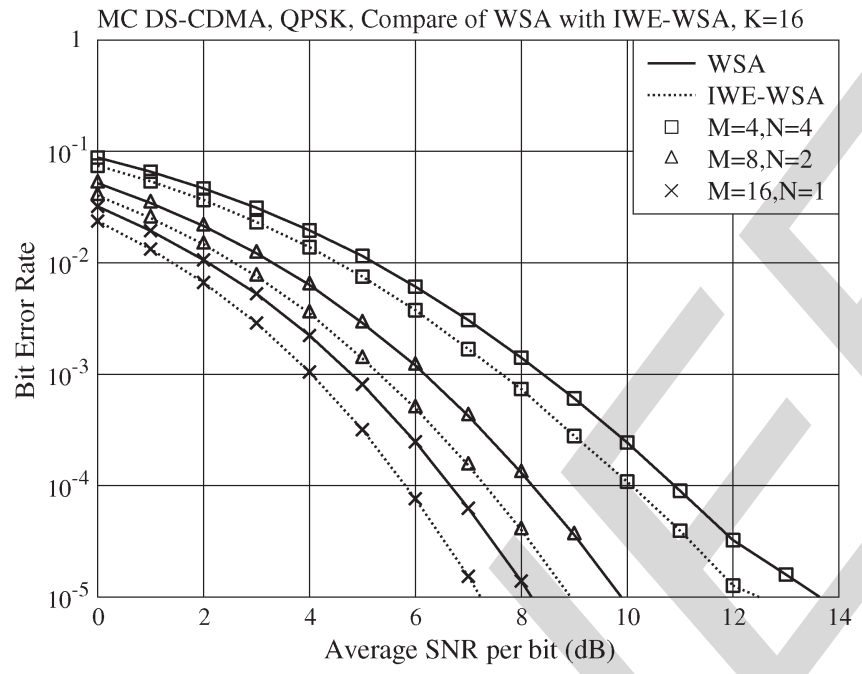

Fig. 9. BER comparison of the downlink MC DS-CDMA systems employing the WSA and the IWE-WSA algorithms, when subcarriers experience independent Rayleigh fading.

920 observe that the improvement of using the IWE scheme for 921 the WCF and the WCA algorithms gets larger as the number 922 of subcarriers $M$ becomes bigger. By contrast, in Fig. 9, the 923 BER advantage of using the IWE remains the same, which is 924 about $1 \mathrm{~dB}$, as the number of subcarriers $M$ becomes bigger. As 925 discussed in Section V, the WE process of the IWE-WCA and 926 IWE-WCF algorithms excludes the worst subcarrier for each 927 user during an iteration, but the worst user of each subcarrier is 928 eliminated during every iteration for the IWE-WSA algorithm. 929 Therefore, the BER performance of the IWE-WCF and IWE930 WCA algorithms is highly affected by the subcarrier diversity, 931 whereas that of the IWE-WSA algorithm is dominated by the 932 user diversity. In Fig. 9, the number of users is $K=16$ for all 933 cases, thus they obtain a similar BER gain when employing the 934 IWE algorithm.

935 So far, we have assumed that all subcarriers of a MC DS936 CDMA system experience independent fading, regardless of the 937 number of subcarriers. When given the frequency selectivity

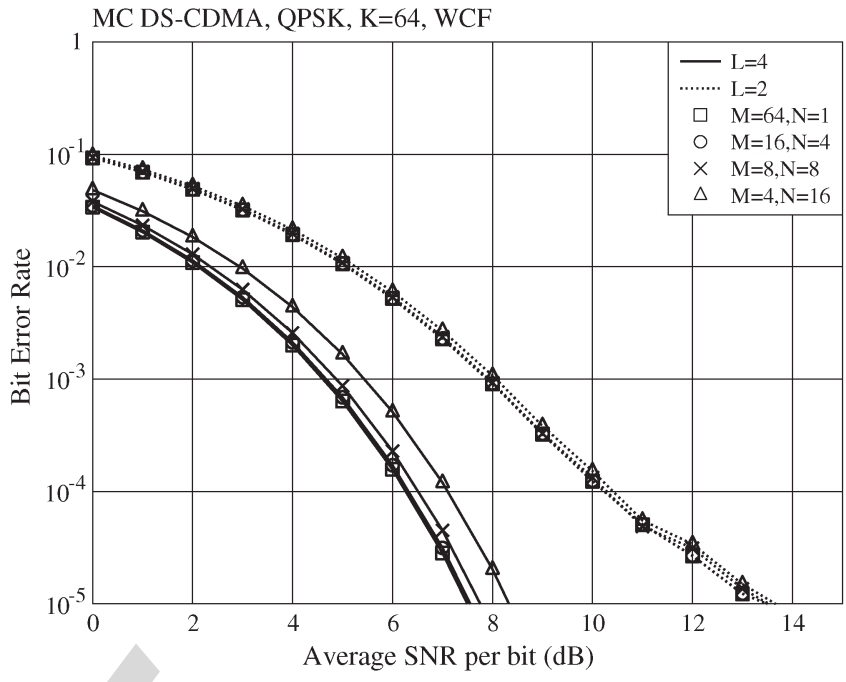

Fig. 10. BER of the downlink MC DS-CDMA systems employing the WCF algorithm, when subcarriers experience frequency selective Rayleigh fading with $L$ number of time domain resolvable paths.

of a wireless channel, this assumption may not be true. In 938 this case, the fading experienced by different subcarriers in 939 fact becomes more correlated, as the number of subcarriers 940 increases. Therefore, in Fig. 10, we study the BER performance 941 of the MC DS-CDMA employing the WCF algorithm, when the 942 number of time-domain resolvable paths is fixed to $L=2$ or 4,943 i.e., when given the frequency selectivity of wireless chan- 944 nels. Explicitly, when $L=2$, using $M=4$ subcarriers is suf- 945 ficient for attaining all the frequency diversity. By contrast, 946 when $L=4, M=16$ subcarriers are required to achieve all 947 the frequency diversity.

\section{CONCLUSION}

949

We have proposed a range of fair subcarrier-allocation al- 950 gorithms and investigated them in the context of the MC DS- 951 CDMA, where the number of users supported may be higher 952 than the number of subcarriers. By analyzing the characteristics 953 of the WSA algorithm that is beneficial to the systems with 954 subcarriers more than users, we have generalized the WSA 955 algorithm to the WCA algorithm, which is suitable for any 956 multicarrier systems. Following our detailed analysis of these 957 algorithms, we have proposed the WCF algorithm, which is 958 capable of further improving the reliability of MC DS-CDMA 959 systems. Moreover, an IWE algorithm has been proposed for 960 application in conjunction with the WSA, WCA or the WCF, 961 resulting in the IWE-WSA, IWE-WCA or the IWE-WCF algo- 962 rithm. Our studies show that an IWE-assisted algorithm always 963 improves the reliability of the original algorithm. The IWE- 964 WCA algorithm outperforms the IWE-WSA algorithm, while 965 the IWE-WCF algorithm achieves the highest reliability among 966 these three. Furthermore, our results demonstrate that the re- 967 liability attained by these IWE-WCF algorithms is close to 968 that achieved by the high-complexity optimum Hungarian algo- 969 rithm. Additionally, the complexity of the proposed subcarrier- 970 allocation algorithms has been analyzed and compared with that 971 of the low-complexity greedy algorithm. We can argue that all 972 
973 our proposed subcarrier-allocation algorithms have the merit of 974 low-complexity.

975 Note that, the observations obtained from this paper are in 976 general suitable for the MC DS-CDMA systems, where dif977 ferent users are allocated with different numbers of subcarriers 978 or/and spreading codes. This is because the relative advantages 979 and disadvantages of the considered subcarrier-allocation algo980 rithms are only determined by the diversity available from the 981 channel quality matrix, i.e., by the values of $K$ and $M$, but not 982 by the numbers of data streams of the users.

\section{REFERENCES}

1] L.-L. Yang, Multicarrier Communications. Chichester, U.K.: Wiley, 2009.

2] L.-L. Yang and L. Hanzo, "Multicarrier DS-CDMA: A multiple access scheme for ubiquitous broadband wireless communications," IEEE Commun. Mag., vol. 41, no. 10, pp. 116-124, Oct. 2003.

3] K. Letaief and Y. J. Zhang, "Dynamic multiuser resource allocation and adaptation for wireless systems," IEEE Trans. Wireless Commun., vol. 13, no. 4, pp. 38-47, Aug. 2006.

[4] J. Jang and K. B. Lee, "Transmit power adaptation for multiuser OFDM systems," IEEE J. Sel. Areas Commun., vol. 21, no. 2, pp. 171-178, Feb. 2003.

[5] K. Kim and J. Kim, "A 2-D subcarrier allocation scheme for capacity enhancement in a clustered OFDM system," IEICE Trans. Commun., vol. E90-B, no. 7, pp. 1880-1883, Jul. 2007.

6] K. A. D. Teo, Y. Otani, and S. Ohno, "Adaptive subcarrier allocation for multi-user OFDM system," IEICE Trans. Commun., vol. E89-A, no. 11, pp. 3131-3137, Nov. 2006.

[7] T. Liu, C. Yang, and L.-L. Yang, "A low-complexity subcarrier-power allocation scheme for frequency-division multiple-access systems," IEEE Trans. Wireless Commun., vol. 9, no. 5, pp. 1564-1570, May 2010.

[8] D. Palomar, J. Cioffi, and M. Lagunas, "Joint Tx-Rx beamforming design for multicarrier MIMO channels: A unified framework for convex optimization," IEEE Trans. Signal Process., vol. 51, no. 9, pp. 2381-2401, Sep. 2003.

9] N. Y. Ermolova and B. Makarevitch, "Performance of practical subcarrier allocation schemes for OFDM," in Proc. IEEE 18th Int. Symp. PIMRC, Sep. 2007, pp. 1-4.

10] O. Nwamadi, X. Zhu, and A. Nandi, "Dynamic subcarrier allocation for single carrier-FDMA systmes," in Proc. EUSIPCO, Aug. 2008, pp. 1-5.

11] H. W. Kuhn, "The Hungarian method for the assignment problem," Naval Res. Logistics Quart., vol. 2, no. 1-2, pp. 83-97, Mar. 1995.

12] T. Jia and A. Duel-Hallen, "Improved channel allocation for multicarrier CDMA with adaptive frequency hopping and multiuser detection," IEEE Trans. Commun., vol. 57, no. 11, pp. 3389-3396, Nov. 2009.

13] Z. Wang, D. Yang, and L. Milstein, "Multi-user resource allocation for a distributed multi-carrier DS-CDMA network," IEEE Trans. Commun., vol. 60, no. 1, pp. 143-152, Jan. 2012.

4] P.-W. Fu and K.-C. Chen, "Rate, sub-carrier, power allocations for multicarrier CDMA with LMMSE multiuser detection," IEEE Trans. Wireless Commun., vol. 6, no. 5, pp. 1574-1580, May 2007.

15] Y. Wang, G. Xie, Y. Liu, and N. Li, "Feasible resource allocation with partial channel information for grouped MC-CDMA system," in Proc. ISCIT, Oct. 2007, pp. 683-688.
[16] E. Lo et al., "Adaptive resource allocation and capacity comparison 1027 of downlink multiuser MIMO-MC-CDMA and MIMO-OFDMA," IEEE 1028 Trans. Wireless Commun., vol. 6, no. 3, pp. 1083-1093, Mar. 2007.

[17] P. Chan et al., "Performance comparison of downlink multiuser MIMO- 1030 OFDMA and MIMO-MC-CDMA with transmit side information- 1031 Multi-cell analysis," IEEE Trans. Wireless Commun., vol. 6, no. 6, 1032 pp. 2193-2203, Jun. 2007.

1033

[18] J. Zhu and Y. Bar-Ness, "Power allocation algorithm in MC-CDMA," in 1034 Proc. IEEE ICC, 2002, vol. 2, pp. 931-935.

1035

[19] Y. Liang, V. Veeravalli, and H. Poor, "Resource allocation for wireless fad- 1036 ing relay channels: Max-Min solution," IEEE Trans. Inf. Theory, vol. 53, 1037 no. 10 , pp. 3432-3453, Oct. 2007.

1038

[20] C. Y. Ng and C. W. Sung, "Low complexity subcarrier and power allo- 1039 cation for utility maximization in uplink OFDMA systems," IEEE Trans. 1040 Wireless Commun., vol. 7, no. 5, pp. 1667-1675, May 2008.

[21] J. Munkres, "Algorithms for the assignment and transportation problems," 1042 J. Soc. Ind. Appl. Math., vol. 5, no. 1, pp. 32-38, Mar. 1957.

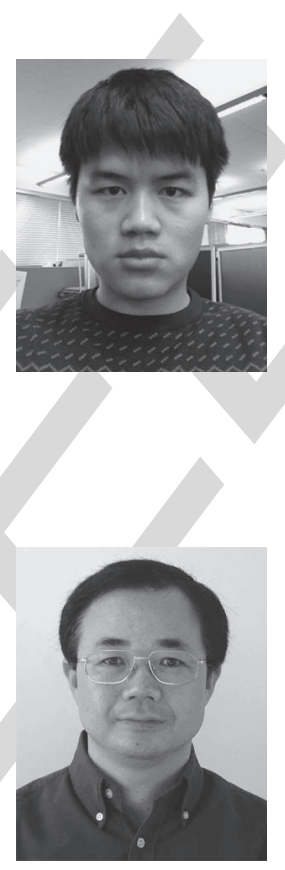

Jia Shi $\left(S^{\prime} X X\right)$ received the B.E. degree (with 1044 AQ1 first-class honors) in electronic and electrical engi- 1045 neering from the University of Central Lancashire, 1046 Lancashire, U.K., in 2009 and the M.Sc. degree 1047 (with distinction) in wireless communications from 1048 the University of Southampton, Southampton, U.K., 1049 in $2010 . \mathrm{He}$ is currently working toward the Ph.D. 1050 degree with the University of Southampton. His 1051 research interests include cooperative communica- 1052 tions, resource allocation in single- and multicell 1053 multicarrier systems, and cognitive radios. of Southampton, Southampton, U.K., where he is a Professor of wireless 1066 communications in the School of Electronics and Computer Science. He has 1067 published over 300 research papers in journals and conference proceedings, 1068 authored/coauthored three books, and published several book chapters. 1069 The details about his publications can be found at http://www-mobile.ecs. 1070 soton.ac.uk/lly/. His research has covered a wide range of topics in wireless 1071 communications, networking, and signal processing. Dr. Yang is a Fellow 1072 of the IET. He served as an Associate Editor of the IEEE TRANSACTIONS 1073 ON VEHICULAR TECHNOLOGY and the Journal of Communications and 1074 Networks and is currently an Associate Editor of the IEEE ACCESS and the 1075 Security and Communication Networks Journal. 


\section{AUTHOR QUERIES}

\section{AUTHOR PLEASE ANSWER ALL QUERIES}

AQ1 = Please provide membership year.

\section{END OF ALL QUERIES}

